\title{
Can a matter-dominated model with constant bulk viscosity drive the accelerated expansion of the universe?
}

\author{
Arturo Avelino and Ulises Nucamendi \\ Instituto de Física y Matemáticas \\ Universidad Michoacana de San Nicolás de Hidalgo \\ Edificio C-3, Ciudad Universitaria, CP. 58040 \\ Morelia, Michoacán, México \\ E-mail: avelino@ifm.umich.mx, ulises@ifm.umich.mx
}

\begin{abstract}
We test a cosmological model which the only component is a pressureless fluid with a constant bulk viscosity as an explanation for the present accelerated expansion of the universe. We classify all the possible scenarios for the universe predicted by the model according to their past, present and future evolution and we test its viability performing a Bayesian statistical analysis using the SCP "Union" data set (307 SNe Ia), imposing the second law of thermodynamics on the dimensionless constant bulk viscous coefficient $\tilde{\zeta}$ and comparing the predicted age of the universe by the model with the constraints coming from the oldest globular clusters.

The best estimated values found for $\tilde{\zeta}$ and the Hubble constant $H_{0}$ are: $\tilde{\zeta}=$ $1.922 \pm 0.089$ and $H_{0}=69.62 \pm 0.59(\mathrm{~km} / \mathrm{s}) \mathrm{Mpc}^{-1}$ with a $\chi_{\min }^{2}=314\left(\chi_{\text {d.o.f }}^{2}=1.031\right)$. The age of the universe is found to be $14.95 \pm 0.42$ Gyr. We see that the estimated value of $H_{0}$ as well as of $\chi_{\text {d.o.f }}^{2}$ are very similar to those obtained from $\Lambda$ CDM model using the same SNe Ia data set. The estimated age of the universe is in agreement with the constraints coming from the oldest globular clusters. Moreover, the estimated value of $\tilde{\zeta}$ is positive in agreement with the second law of thermodynamics (SLT).

On the other hand, we perform different forms of marginalization over the parameter $H_{0}$ in order to study the sensibility of the results to the way how $H_{0}$ is marginalized. We found that it is almost negligible the dependence between the best estimated values of the free parameters of this model and the way how $H_{0}$ is marginalized in the present work.

Therefore, this simple model might be a viable candidate to explain the present acceleration in the expansion of the universe.

PACS numbers: 95.36.+x, 98.80.-k, 98.80.Es
\end{abstract}

Submitted to: Journal of Cosmology and Astroparticle physics 


\section{Introduction.}

In the last ten years the observations of type Ia supernovae (SNe Ia) have suggested a possible late time accelerated expansion of the universe (see for instance [1]-[7] and references therein). This discovery has additional support from the cosmic microwave background [8] and the large scale structure [9] observations.

Several models have been proposed to explain this recent acceleration, one of them is the assumption of the existence of the so-called dark energy as responsible of such acceleration, i.e., a new unknown component that must be $\sim 70 \%$ of the total content of matter and energy in the universe [1]-[7]. The leading dark energy candidates are a cosmological constant and a slowly varying rolling scalar field (quintessence models) [10]-[14]. However, a cosmological constant model faces several strong problems, one of them is the huge discrepancy between its predicted and observed value (of about 120 orders of magnitude) [15]-[18], another one is the so-called the "cosmic coincidence problem", i.e., why are we living in a moment where the matter density in the universe is of the same order than the dark energy density? [19]-[21].

On the other hand, it has been known since several years ago before of the discovery of the present acceleration in the expansion of the universe that a bulk viscous fluid can produce an accelerating cosmology (although it was originally proposed in the context of an inflationary period in the early universe) without the need of any cosmological constant or dark energy component [22]-[35] although some authors do not agree with this conclusion [36].

So, it is natural to think of the bulk viscous pressure as one of the possible mechanism that can accelerate the universe today (see for instance [37]-[46], where in some of these works the bulk viscous coefficient is assumed a priori without being derived from known physics or known particle properties). However, this idea faces the problem of that it is necessary to propose a viable mechanism for the origin of the bulk viscosity and in this sense some proposals have been already suggested [47]-[50].

Nowadays, with the observational data sets of SNe Ia [1]-[7] and some other cosmological observations like the shift parameter $R$ of the cosmic microwave background radiation (CMB) given by the Wilkinson Microwave Anisotropy Probe (WMAP) observations [51], and the baryon acoustic oscillation (BAO) measurement from the Sloan Digital Sky Survey (SDSS) [52] it is possible to test cosmological models. So, in the present work we study and test bulk viscous matter-dominated cosmologies, i.e., scenarios which the only component of the universe is a pressureless fluid with bulk viscosity. The pressureless fluid characterizes to both baryon and dark matter components. The idea of this model is to explain the present acceleration of the universe using the bulk viscous pressure of the fluid without the need of any dark energy.

As it was mentioned above, the explicit form of the bulk viscosity has to be assumed a priori or obtained from a known physical mechanism. In the present work we choose the first possibility. So, we assume a constant bulk viscous coefficient that it is the simplest parametrization for the bulk viscosity and we estimate its value using SNe Ia 
observations. The theoretical solution of this model has been also analyzed in [22], [53]-[56] and other parametrizations for the bulk viscous coefficient $\zeta$ have been also proposed and studied in [37]-[45] and [57]-[60]. There is also other general approach to the bulk viscous cosmologies called "fluids with inhomogeneous equation of state" (see $[61,65])$.

In section 2 we present the generalities of bulk viscous fluids in General Relativity (GR), in section 3 we apply this formalism to a bulk viscous matter-dominated universe model where we find the explicit expression for the Hubble parameter in function of the redshift. In section 4 we analyze the behavior of the scale factor for the possible scenarios that the model predicts for the universe according to the value of the bulk viscous coefficient. In section 5 we study the behavior of the deceleration parameter $q$, the curvature scalar $R$, the total matter density $\rho_{\mathrm{m}}$ and the age of the universe and in section 6 we briefly review the second law of thermodynamics (SLT). Section 7 presents the SNe Ia test to constrain the model and compute the best estimated values for the bulk viscous coefficient and the Hubble constant. After that, section 8 presents the best estimated values and the probability distribution functions for the bulk viscous coefficient by marginalizing over the Hubble constant assuming three different priors and finally in section 9 we present our conclusions. The appendices A, B and C detail the marginalization methods used in the present work.

\section{Theory of relativistic bulk viscous fluids.}

The origin of the bulk viscosity in a physical system is due to its deviations from the local thermodynamic equilibrium (for a review in the theory of relativistic dissipative fluids see [66]). In a cosmological fluid, the bulk viscosity arises when the fluid expands (or contracts) too fast so that the system does not have enough time to restore the local thermodynamic equilibrium and then it appears an effective pressure restoring the system to its local thermodynamic equilibrium. The bulk viscosity can be seen as a measurement of this effective pressure. When the fluid reaches again the thermal equilibrium then the bulk viscosity pressure ceases [49], [67]-[69].

In an accelerated expanding universe it is very possible that the expansion process is actually a collection of states out of thermal equilibrium in a small fraction of time. So, it is natural to assume the existence of a bulk viscous coefficient in a more realistic description of the accelerated universe today.

We use the Weinberg formalism [70]-[72] for the imperfect fluids. So, in the present work we consider a bulk viscous fluid as source of matter in the Einstein fields equations $G_{\mu \nu}=8 \pi G T_{\mu \nu}$, where $G$ is the Newton gravitational constant.

On the other hand, the energy-momentum tensor of an imperfect fluid with a firstorder deviation from the thermodynamic equilibrium has the form [49, 70, 71]:

$$
T_{\mu \nu}=\rho u_{\mu} u_{\nu}+\left(g_{\mu \nu}+u_{\mu} u_{\nu}\right) P^{*}
$$


where

$$
P^{*} \equiv P-\zeta \nabla^{\nu} u_{\nu}
$$

In the equations (1) and (2) the four-velocity vector $u_{\nu}$ is of an observer who measures the effective pressure $P^{*}, P$ and $\rho$ are the pressure and density of the fluid respectively. The term $\zeta$ is the bulk viscous coefficient that arises in the fluid which is out of the local thermodynamic equilibrium.

It can be seen that the energy-momentum tensor (1) is similar to that of a perfect fluid but with an effective pressure $P^{*}$ composed by the usual pressure $P$ of the fluid plus the pressure due to its bulk viscosity $P_{\text {visc }} \equiv-\zeta \nabla^{\nu} u_{\nu}$. This viscous pressure, $P_{\text {visc }}$, can be seen as a "measurement" of the pressure to restore the local thermodynamic equilibrium [49], [67]-[69]. The conservation equation for the viscous fluid is

$$
u^{\nu} \nabla_{\nu} \rho+\left(\rho+P^{*}\right) \nabla^{\nu} u_{\nu}=0
$$

The effective pressure (2) was originally proposed by Eckart [74] in 1940 for a relativistic dissipative process in the context of thermodynamics systems out of local equilibrium, and subsequently Landau \& Lifshitz presented an equivalent formulation [75].

However, the Eckart theory has problems in several aspects. One of them is that all the equilibrium states in this theory are unstable [76]. Another one is that signals can propagate through the fluids with superluminal velocities [77, 78].

In 1979, Israel-Stewart [79, 80] developed a more consistent and general theory that avoids these problems, and from which the Eckart theory is the first-order limit of the Israel-Stewart theory when the relaxation time goes to zero. Nevertheless, the Eckart theory is simpler to deal with than the Israel-Stewart theory.

Despite of the inherent problems of the Eckart theory, and due to that it is simpler than the Israel-Stewart theory, it has been widely used recently by several authors to model bulk viscous dark fluids as responsible of the recent observed acceleration of the universe assuming that the approximation is valid for this purpose (see, for instance [37]-[41], [44, 45, 49, 56, 60]), i.e., in these papers assume a vanishing relaxation time, so that, in this limit the Eckart theory is a good approximation to the Israel-Stewart theory. In this context, it is convenient to mention that Hiscock et al [36] showed that flat Friedmann-Robertson-Walker cosmological models containing a bulk viscous Boltzmann gas expand more rapidly using the Eckart theory than the Israel-Stewart one, and suggesting that inflationary acceleration driven by bulk viscosity could be an effect of applying a pathological theory such as the Eckart theory. These results suggest that in this context the use of the Israel-Stewart causal theory would not produce a recent accelerating epoch as the Eckart theory would. However, posterior studies have suggested that this conclusion could not be true because consistent inflationary solutions have been found using the Israel-Stewart theory [34, 35].

It is important to point out that there exists other more general formulation for irreversible processes than the Israel-Stewart theory developed by D. Pavón et al. where the temperature is not necessary that of the thermodynamic equilibrium, see [81] for 
details. In the present work we assume the Eckart theory and we constrain the model described above using SNe Ia observations.

\section{Cosmological model of bulk viscous matter-dominated universes.}

We study a cosmological model in a flat universe where the only component is a pressureless fluid with constant bulk viscosity as an explanation for the present accelerated expansion of the universe. The pressureless fluid characterizes both the baryon and dark matter components.

Note that this model does not have the Cosmic Coincidence problem and that this fluid represents an unified description of the dark sector plus the baryon component in a similar way than the Chaplygin gas model (see for instance [44, 82] and references therein). In this approach the present acceleration of the universe is driven by the bulk viscous pressure of the fluid instead of a dark energy component. The present work differs from the approach of Colistete et al [44] in that they propose two fluids, one of them is a bulk viscous fluid representing in an unified way the dark sector with a bulk viscous coefficient $\zeta$ proportional to a power of the energy density $\rho$ (i.e., $\zeta=\tilde{\zeta} \rho^{\nu}$, with $\tilde{\zeta}, \nu$ constants), and the other one is a pressureless fluid representing the baryon component. Nevertheless, most of their analysis and test using cosmological observations is done using the ansatz $\nu=0$.

Since we work with a pressureless fluid $(P=0)$ then $P^{*}=P_{\text {visc }} \equiv-\zeta \nabla^{\nu} u_{\nu}$, where $\zeta$ is the bulk viscous coefficient of the matter fluid.

We consider a spatially flat geometry for the Friedmann-Robertson-Walker (FRW) cosmology as favored by WMAP [83]

$$
d s^{2}=-d t^{2}+a^{2}(t)\left(d r^{2}+r^{2} d \Omega^{2}\right)
$$

where the function $a(t)$ is the scale factor. On the other hand, we spread the conservation equation (3) in all its components

$$
\dot{\rho}_{\mathrm{m}}+\left(\rho_{\mathrm{m}}-3 H \zeta\right) 3 H=0
$$

where $H \equiv \dot{a} / a$ is the Hubble parameter, $\rho_{\mathrm{m}}$ is the total matter density, the dot means time derivative and $\nabla^{\nu} u_{\nu}=3 H$.

The conservation equation (5) can be written in terms of the scale factor as

$$
a \frac{d \rho_{\mathrm{m}}}{d a}=3\left(3 H \zeta-\rho_{\mathrm{m}}\right)
$$

This equation is valid for any parametrization of $\zeta$, in particular we assume the ansatz $\zeta=$ constant that it is perhaps the simplest parametrization for the bulk viscous coefficient that can be proposed. Its value is going to be estimated from the SNe Ia observations.

On the other hand, the first Friedmann equation for a flat universe is

$$
H^{2}=\frac{8 \pi G}{3} \rho_{\mathrm{m}}
$$


So, substituting (7) into (6) we obtain

$$
a \frac{d \rho_{\mathrm{m}}}{d a}+3 \rho_{\mathrm{m}}-\gamma \rho_{\mathrm{m}}^{1 / 2}=0, \quad \text { where } \quad \gamma \equiv 9\left(\frac{8 \pi G}{3}\right)^{1 / 2} \zeta
$$

Changing of variable from the scale factor to the redshift $z$ using the relationship $\neq$ $a=1 /(1+z)$, we obtain the ordinary differential equation (ODE):

$$
(1+z) \frac{d \rho_{\mathrm{m}}}{d z}-3 \rho_{\mathrm{m}}+\gamma \rho_{\mathrm{m}}^{1 / 2}=0
$$

The exact solution of this ODE is:

$$
\rho_{\mathrm{m}}(z)=\left[\frac{\gamma}{3}+\left(\rho_{\mathrm{m} 0}^{1 / 2}-\frac{\gamma}{3}\right)(1+z)^{3 / 2}\right]^{2}
$$

where $\rho_{\mathrm{m} 0}$ is the matter density evaluated today. Substituting this solution into (7) we obtain

$$
H^{2}(z)=H_{0}^{2}\left[\frac{\tilde{\zeta}}{3}+\left(\Omega_{\mathrm{m} 0}^{1 / 2}-\frac{\tilde{\zeta}}{3}\right)(1+z)^{3 / 2}\right]^{2}
$$

where $H_{0}$ is the Hubble constant and where we have defined the dimensionless bulk viscous coefficient $\tilde{\zeta}$, the matter density parameter $\Omega_{\mathrm{m} 0}$ and the critical density today $\rho_{\text {crit }}^{0}$ as:

$$
\tilde{\zeta} \equiv \frac{24 \pi G}{H_{0}} \zeta, \quad \Omega_{\mathrm{m} 0} \equiv \frac{\rho_{\mathrm{m} 0}}{\rho_{\text {crit }}^{0}}, \quad \rho_{\text {crit }}^{0} \equiv \frac{3 H_{0}^{2}}{8 \pi G}
$$

In this model the bulk viscous matter is the only component of the universe implying that the first Friedmann equation (7) evaluated today is $\Omega_{\mathrm{m} 0}=1$. With this, the expression (11) finally becomes

$$
H(z)=\frac{H_{0}}{3}\left[\tilde{\zeta}+(3-\tilde{\zeta})(1+z)^{3 / 2}\right]
$$

\section{Classification and evolution of bulk viscous matter-dominated models.}

In this section we classify all the possibilities for the universe predicted by this bulk viscous matter-dominated model using different values of the constant bulk viscous coefficient. Note that we have one different model for each value of $\tilde{\zeta}$, so actually we have a collection of models depending of the value of $\tilde{\zeta}$.

We analyze the theoretical behavior of the scale factor in terms of the cosmic time. We begin expressing (13) in terms of the scale factor

$$
H(a) \equiv \frac{\dot{a}}{a}=\frac{H_{0}}{3}\left(\frac{\tilde{\zeta} a^{3 / 2}+3-\tilde{\zeta}}{a^{3 / 2}}\right)
$$

Integrating (14) yields

$$
H_{0}\left(t-t_{0}\right)=H_{0} \int_{t_{0}}^{t} d t^{\prime}=3 \int_{1}^{a} \frac{a^{1 / 2} d a^{\prime}}{\tilde{\zeta} a^{\prime 3 / 2}+3-\tilde{\zeta}}
$$

where $t$ labels the cosmic time and $t_{0}$ the cosmic time today.

$\ddagger$ We assume that the value of the scale factor evaluated today is equal to one. 
4.1. Case $\tilde{\zeta}=0$.

When $\tilde{\zeta}=0$ we recover the usual matter-dominated universe (with null bulk viscosity) with a scale factor coming from the integration of (15) like:

$$
a(t)=\left(\frac{3}{2} H_{0}\left(t-t_{0}\right)+1\right)^{2 / 3}, \quad \tilde{\zeta}=0
$$

The first and second derivatives of the scale factor with respect to $x \equiv H_{0}\left(t-t_{0}\right)$ are

$$
\frac{d a}{d x}=\left(\frac{2}{3 x+2}\right)^{1 / 3}
$$

and

$$
\frac{d^{2} a}{d x^{2}}=-\frac{2^{1 / 3}}{(3 x+2)^{4 / 3}}
$$

The plot of functions (16)-(18) are shown in figures 1-3 respectively (the long dashed lines).

This case predicts an eternal decelerated expanding universe (see figures $1-7$ ). In this case the curvature scalar and the matter density are $R=\left(3 H_{0}^{2}\right) a^{-3}$ and $\rho_{\mathrm{m}}=$ $\left(3 H_{0}^{2} / 8 \pi G\right) a^{-3}$ respectively, that both diverge when the scale factor goes to zero (see sections 5.2 and 5.3 for details). When we have these two conditions $(R(a \rightarrow 0) \rightarrow \infty$ and $\left.\rho_{\mathrm{m}}(a \rightarrow 0) \rightarrow \infty\right)$ in the past of the universe we say that there was a Big-Bang. The elapsed time between the Big-Bang time till today is

$$
t_{\mathrm{B}}=t_{0}-\frac{2}{3 H_{0}}
$$

where the subscript 'B' stands for "Big-Bang". On the other hand, when $a \rightarrow \infty$ then $R$ and $\rho_{\mathrm{m}}$ decrease to zero.

\section{2. $\operatorname{Case} \tilde{\zeta} \neq 0$.}

For $\tilde{\zeta} \neq 0$ we do the change of variable $y \equiv a^{3 / 2}$ in expression (15) yielding:

$$
H_{0}\left(t-t_{0}\right)=\frac{2}{\tilde{\zeta}} \int_{1}^{y} \frac{\tilde{\zeta} d y^{\prime}}{\tilde{\zeta} y^{\prime}+3-\tilde{\zeta}}=\frac{2}{3 \tilde{\zeta}} \ln \left|\tilde{\zeta} a^{3 / 2}+3-\tilde{\zeta}\right|
$$

This last expression can be rewritten as

$$
3 \exp \left[\frac{\tilde{\zeta}}{2} H_{0}\left(t-t_{0}\right)\right]=\left|\tilde{\zeta} a^{3 / 2}+3-\tilde{\zeta}\right|
$$

When $\tilde{\zeta} a^{3 / 2}+3-\tilde{\zeta} \geq 0$ we can remove the absolute value bar of the right hand side term and then we obtain

$$
a(t)=\left[\frac{3 \exp \left(\frac{1}{2} \tilde{\zeta} H_{0}\left(t-t_{0}\right)\right)-3+\tilde{\zeta}}{\tilde{\zeta}}\right]^{2 / 3}, \quad \tilde{\zeta} \neq 0
$$


The first and second derivative of the scale factor with respect to $x \equiv H_{0}\left(t-t_{0}\right)$ are

$$
\frac{d a}{d x}=\left[\frac{\tilde{\zeta} \exp \left(\frac{3}{2} \tilde{\zeta} x\right)}{3 \exp \left(\frac{3}{2} \tilde{\zeta} x\right)+\tilde{\zeta}-3}\right]^{1 / 3}
$$

and

$$
\frac{d^{2} a}{d x^{2}}=\frac{\tilde{\zeta}^{4 / 3} \exp \left(\frac{1}{2} \tilde{\zeta} x\right)\left[2 \exp \left(\frac{1}{2} \tilde{\zeta} x\right)+\tilde{\zeta}-3\right]}{2\left[3 \exp \left(\frac{1}{2} \tilde{\zeta} x\right)+\tilde{\zeta}-3\right]^{4 / 3}}
$$

The behavior of the expressions (22)-(24) are shown in figures 1-3 respectively.

The case $\tilde{\zeta} a^{3 / 2}+3-\tilde{\zeta}<0$ does not correspond to a physical case of our interest because this case predicts an eternal contraction of the universe $(H(t)<0$ for any value of the cosmic time $t$ ) that is in contradiction with the observations (see the expression (14) to note that $\tilde{\zeta} a^{3 / 2}+3-\tilde{\zeta}<0$ implies $\left.H(t)<0\right)$.

In order to study carefully the expression (22) for different values of $\tilde{\zeta}$ we take four cases:

- $0<\tilde{\zeta}<3$

- $\tilde{\zeta}=3$

- $\tilde{\zeta}>3$

- $\tilde{\zeta}<0$

4.2.1. Case $0<\tilde{\zeta}<3$. From the expression (22) we can see that when $t \rightarrow \infty$ then the scale factor tends to have the form like that of the de Sitter universe, i.e.,

$$
a(t) \propto \mathrm{e}^{(\tilde{\zeta} / 3) H_{0}\left(t-t_{0}\right)}
$$

On the other hand, for any value of $\tilde{\zeta}$ in this interval all the models predict an universe having a Big-Bang in the past in the cosmic time:

$$
t_{\mathrm{B}}=t_{0}+\frac{2}{\tilde{\zeta} H_{0}} \ln \left(1-\frac{\tilde{\zeta}}{3}\right)
$$

So, the universe begins with a Big-Bang followed by an eternal expansion (there is not any recollapse epoch, see figures 1 and 2) and this expansion begins with a decelerated epoch followed by an eternal accelerated one (see figures 2 and 3).

The transition between the decelerated-accelerated expansion epochs depends on the value of $\tilde{\zeta}$. We compute the value of the scale factor where the transition happens. For that, we derive to $\dot{a}$ with respect to $a$ using the expression (14):

$$
\frac{d \dot{a}}{d a}=\frac{H_{0}}{3}\left(\tilde{\zeta}+\frac{\tilde{\zeta}-3}{2 a^{3 / 2}}\right)
$$




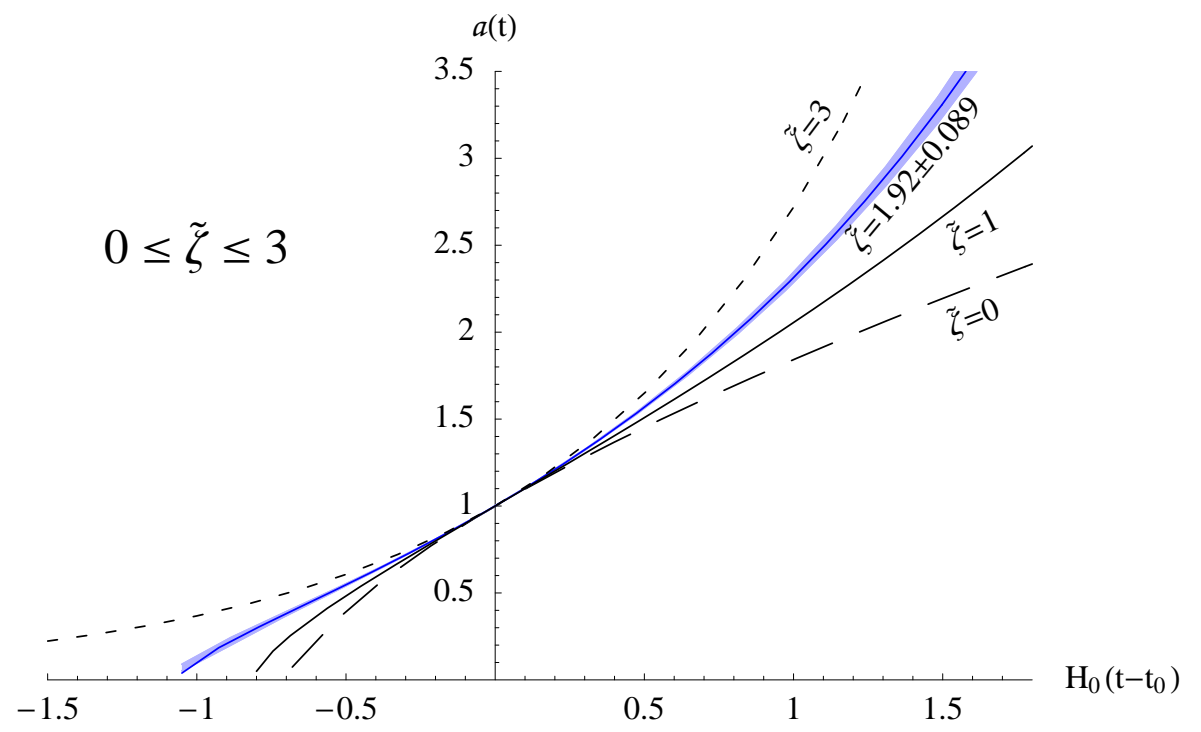

Figure 1. Plot of $a(t, \tilde{\zeta})$ for different values of $\tilde{\zeta}$ in the interval $0 \leq \tilde{\zeta} \leq 3$ (see expressions (16) and (22)). $H_{0}$ is the Hubble constant, $t$ is the cosmic time and $t_{0}$ is its present value. The long dashed line corresponds to $\tilde{\zeta}=0$ (a flat matterdominated universe with null bulk viscosity, see expression (16)). The short dashed line corresponds to $\tilde{\zeta}=3$ (the de Sitter universe). The blue line with the blue band correspond to a model with $\tilde{\zeta}=1.922 \pm 0.089$. This is the best estimated value of $\tilde{\zeta}$ coming from the SCP "Union" SNe Ia data set analysis (see section 7). The band corresponds to the error at the $68.3 \%$ confidence level.

Then, we make equal to zero to expression (27) in order to obtain the value of the scale factor " $a_{\mathrm{t}}$ " where the transition happens, obtaining

$$
a_{\mathrm{t}}=\left(\frac{3-\tilde{\zeta}}{2 \tilde{\zeta}}\right)^{2 / 3}
$$

where the subscript "t" stands for "transition". Writing the expression (28) in terms of the redshift $z$ yields

$$
z_{\mathrm{t}}=\left(\frac{2 \tilde{\zeta}}{3-\tilde{\zeta}}\right)^{2 / 3}-1
$$

From the expression (28) we can see that for values of $\tilde{\zeta}$ in the interval $0<\tilde{\zeta}<1$ the transition between the decelerated epoch to the accelerated one takes place in the future $\left(a_{\mathrm{t}}>1\right)$. When $\tilde{\zeta} \rightarrow 0$, the value of $a_{\mathrm{t}}$ tends to infinity in the future. When $\tilde{\zeta}=1$ then the transition takes place today $\left(a_{\mathrm{t}}=1\right)$, when $1<\tilde{\zeta}<3$ the transition takes place in the past of the universe $\left(0<a_{\mathrm{t}}<1\right)$ and when the value of $\tilde{\zeta}$ is closer to 3 , the transition is closer to the Big-Bang time (see figures 2-4 and 7).

The curvature scalar $R$ is always positive and it has a very large value when the scale factor is very small (at the Big-Bang time) and it decreases forever tending to its minimum value $R=\frac{4}{3} H_{0}^{2} \tilde{\zeta}$ when $a \rightarrow \infty$ (see section 5.2 and figure 8 ) what is the 


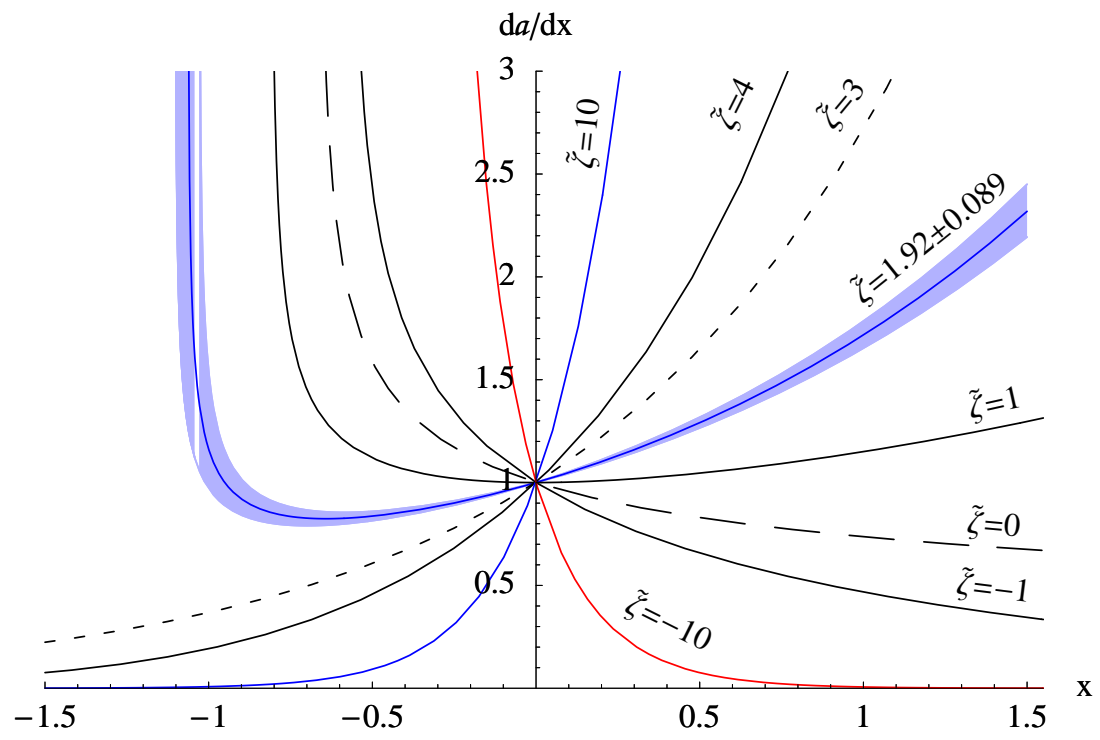

Figure 2. Plot of the derivative of the scale factor with respect to $x \equiv H_{0}\left(t-t_{0}\right)$ for different values of $\tilde{\zeta}$ (see expression (23)). The long dashed line corresponds to $\tilde{\zeta}=0$ (a flat matter-dominated universe with null bulk viscosity, see expression (17)) and the short dashed line corresponds to $\tilde{\zeta}=3$ (the de Sitter universe). The blue line with the blue band correspond to a model with $\tilde{\zeta}=1.922 \pm 0.089$. This is the best estimated value of $\tilde{\zeta}$ coming from the SCP "Union" SNe Ia data set analysis (see section 7 ). The band corresponds to the error at the $68.3 \%$ confidence level.

de Sitter universe (consistent with the expression (25)). In the same way, the matter density $\rho_{\mathrm{m}}$ is also very large when the scale factor is very small (at the Big-Bang time) and it decreases forever tending to its minimum value $\rho_{\mathrm{m}}=\left(H_{0}^{2} / 24 \pi G\right) \tilde{\zeta}^{2}$ when $a \rightarrow \infty$ (see section 5.3).

The possible value for $\tilde{\zeta}$ at least at the $99.9 \%$ confidence level estimated from the SCP "Union" SNe Ia data set is in the interval $1<\tilde{\zeta}<3$. So, assuming the best estimated value $\tilde{\zeta}=1.922 \pm 0.089$ (see table 1 ), the value of the scale factor at the transition time between deceleration-acceleration epochs is (see expression (28)): $a_{\mathrm{t}}=0.42 \pm 0.03 \quad\left(z_{\mathrm{t}}=1.33 \pm 0.2\right)$. Note that the transition comes directly from the model, it is not necessary to assume any particular ansatz to estimate or induce it.

4.2.2. Case $\tilde{\zeta}=3$. In the expression (22) we put $\tilde{\zeta}=3$ to obtain

$$
a(t)=\mathrm{e}^{H_{0}\left(t-t_{0}\right)}
$$

This case corresponds to the de Sitter universe. Figures 1-5 show the behavior of expression (30) (the short-dashed lines). The scale factor becomes zero just when $H_{0}\left(t-t_{0}\right) \rightarrow-\infty$ and when $H_{0}\left(t-t_{0}\right) \rightarrow \infty$ the scale factor increases to infinity.

In this case the model predicts an universe in an eternal accelerated expansion. The curvature scalar and the matter density are constants with values $R=12 H_{0}^{2}$ and $\rho_{\mathrm{m}}=3 H_{0}^{2} / 8 \pi G$ respectively. See sections 5.2 and 5.3 for details. 


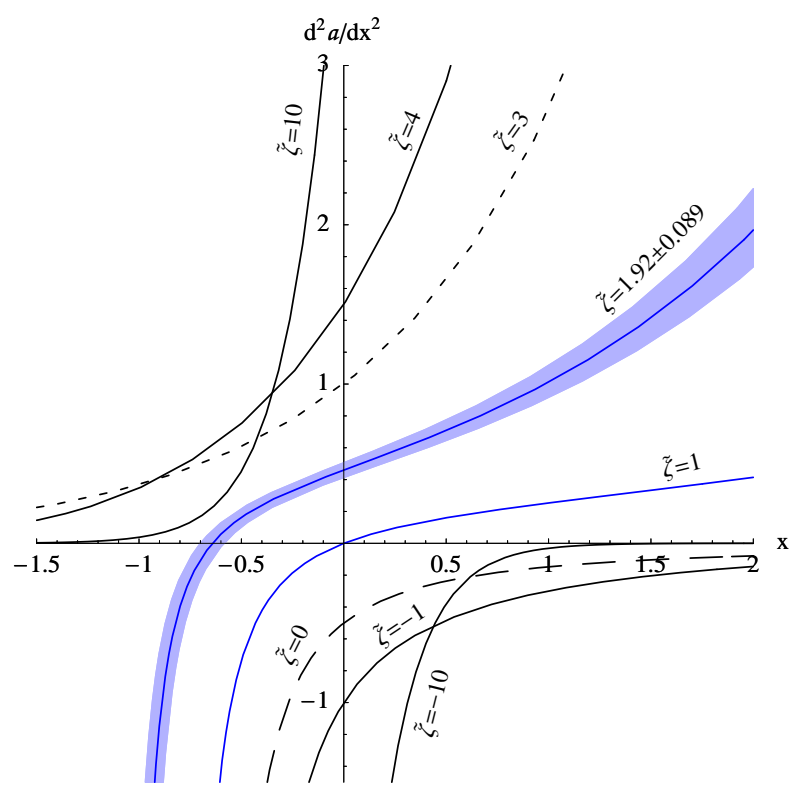

Figure 3. Plot of the second derivative of the scale factor with respect to $x \equiv H_{0}\left(t-t_{0}\right)$ for different values of $\tilde{\zeta}$ (see expression (24)). The long dashed line corresponds to $\tilde{\zeta}=0$ (a flat matter-dominated universe with null bulk viscosity, see expression (18)) and the short dashed line corresponds to $\tilde{\zeta}=3$ (the de Sitter universe). The blue line with the blue band correspond to a model with $\tilde{\zeta}=1.922 \pm 0.089$. This is the best estimated value of $\tilde{\zeta}$ coming from the SCP "Union" SNe Ia data set analysis (see section 7). The band corresponds to the error at the $68.3 \%$ confidence level. We see that for $\tilde{\zeta} \leq 0$ all the models predict a decelerated universe $\left(d^{2} a / d x^{2}<0\right)$ forever. For $\tilde{\zeta} \geq 3$ the models predict an accelerated universe $\left(d^{2} a / d x^{2}>0\right)$ forever. And in the range $0<\tilde{\zeta}<3$ the models predict a transition from deceleration to acceleration.

Note that this model does not have a Big-Bang because the curvature scalar and the matter density are regular for any value of the cosmic time.

4.2.3. Case $\tilde{\zeta}>3$. This case predicts an universe expanding forever, and this expansion is always accelerated (there is not any decelerating epoch or acceleration-deceleration transition).

In this case when $t \rightarrow \infty$ then the scale factor tends to the form of the de Sitter universe in the future (see expression (25)) and when $t \rightarrow-\infty$ then the universe tends to an Einstein static universe (defined by $\dot{a}, \ddot{a}=0$ ) where the value of the scale factor is not zero but tends to its minimum value:

$$
\lim _{t \rightarrow-\infty} a(t) \equiv a^{*}=\left(1-\frac{3}{\tilde{\zeta}}\right)^{2 / 3}
$$

and during the evolution of the universe the scale factor is an increasing monotonic function with respect to the cosmic time. Thus, for this case there was not a Big-Bang and the age of the universe is not defined. Figure 5 shows some plots of the expression (22) for different values of $\tilde{\zeta}>3$. 


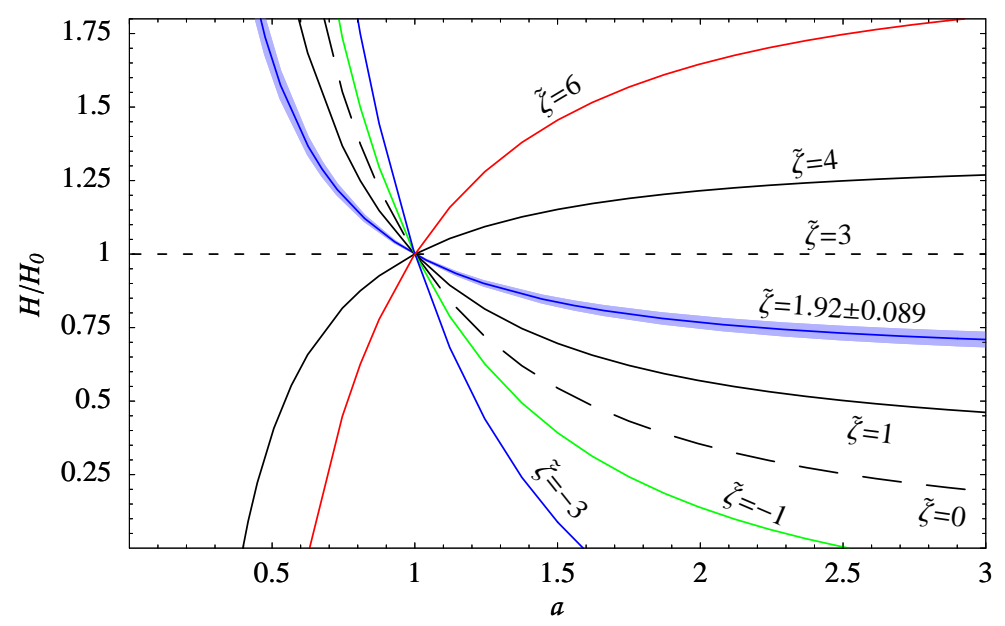

Figure 4. Plot of $H(a, \tilde{\zeta})$ for different values of $\tilde{\zeta}$ (see expression (14)). $a$ is the scale factor and $H_{0}$ is the Hubble constant. The long dashed line corresponds to $\tilde{\zeta}=0$ (a flat matter-dominated universe with null bulk viscosity) and the short dashed line corresponds to $\tilde{\zeta}=3$ (the de Sitter universe). The blue line with the blue band correspond to a model with $\tilde{\zeta}=1.922 \pm 0.089$. This is the best estimated value of $\tilde{\zeta}$ coming from the SCP "Union" SNe Ia data set analysis (see section 7). The band corresponds to the error at the $68.3 \%$ confidence level.

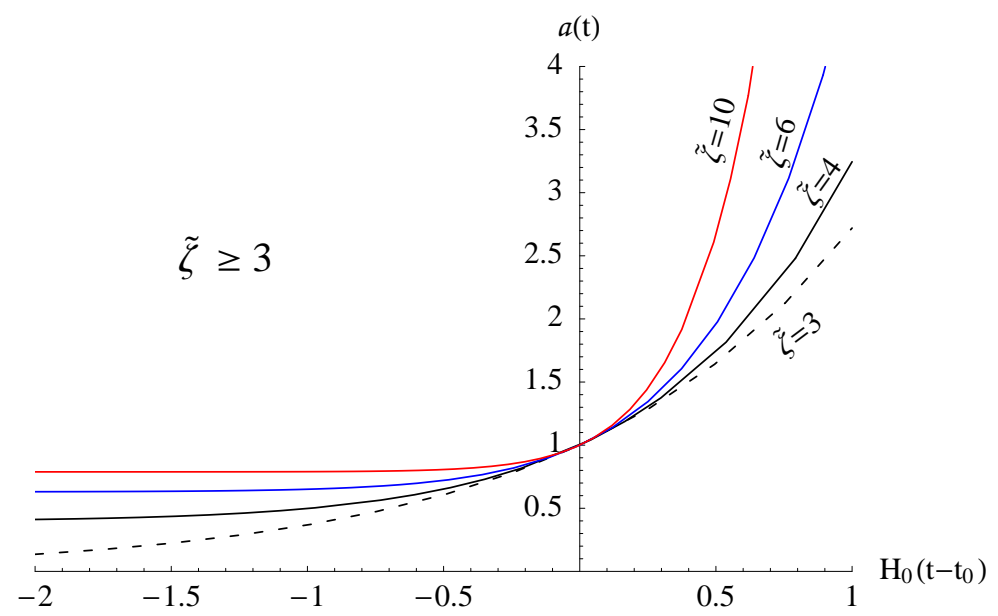

Figure 5. Plot of $a(t, \tilde{\zeta})$ for different values of $\tilde{\zeta}$ in the range $\tilde{\zeta} \geq 3$ (see expression (22)). $H_{0}$ is the Hubble constant, $t$ is the cosmic time and $t_{0}$ is its present value. The short dashed line corresponds to $\tilde{\zeta}=3$ (the de Sitter universe).

When the scale factor has the value $a^{*}$ the curvature scalar is zero and it is always increasing from zero until to reach its maximum value $R=\frac{4}{3} H_{0}^{2} \tilde{\zeta}$ when $a \rightarrow \infty$. See section 5.2 and figure 8 for details.

The matter density $\rho_{\mathrm{m}}$ has a similar behavior to the curvature scalar in the sense of that when the scale factor has the value $a^{*}$ the matter density is zero and it is always increasing from zero until to reach its maximum value $\rho_{\mathrm{m}}=\left(H_{0}^{2} / 24 \pi G\right) \tilde{\zeta}^{2}$ when $a \rightarrow \infty$. See section 5.3 and figure 9 for details. 


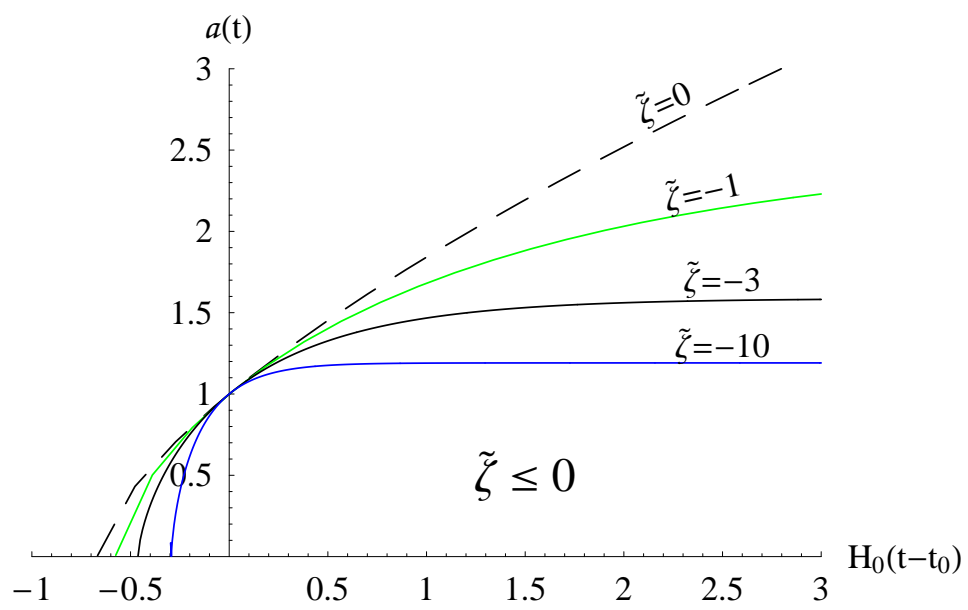

Figure 6. Plot of $a(t, \tilde{\zeta})$ for different values of $\tilde{\zeta}$ in the range $\tilde{\zeta} \leq 0$ (see expressions (16) and (22)). $H_{0}$ is the Hubble constant, $t$ is the cosmic time and $t_{0}$ is its present value. The long dashed line corresponds to $\tilde{\zeta}=0$ (a flat matter-dominated universe with null bulk viscosity, see expression (16)).

4.2.4. Case $\tilde{\zeta}<0$. This case predicts an eternal decelerated expanding universe. The universe begins with a Big-Bang and expands forever until to reach its maximum value $a^{*}=(1-3 / \tilde{\zeta})^{2 / 3}$ when $t \rightarrow \infty$, becoming an Einstein static universe in the future. The Big-Bang took place in the cosmic time $t_{\mathrm{B}}$ defined by expression (26). Figures $2-4$ and 6 show the behavior of the scale factor for this case.

In this case the curvature scalar has a transition from positive to negative values at $a_{0} \equiv[(\tilde{\zeta}-3) / 4 \tilde{\zeta}]^{2 / 3}$. When $a \rightarrow 0$ then $R \rightarrow \infty$ (the Big-Bang) and when $a=a^{*}$ the curvature scalar is zero. The minimum value of the curvature scalar is negative and it is reached at the value of the scale factor $\tilde{a} \equiv[(2-6 / \tilde{\zeta}) / 5]^{2 / 3}$. See section 5.2 and figure 8 for details.

The matter density is always a decreasing function over its evolution starting from infinity (when $a \rightarrow 0$ ) to zero (when the scale factor is $a^{*}$ ). See section 5.3 and figure 9 for details.

\section{Cosmological parameters.}

\subsection{Deceleration parameter $q$.}

We study the behavior of the deceleration parameter $q$ function that is defined as

$$
q(a) \equiv-\frac{\ddot{a} a}{\dot{a}^{2}}=-\frac{\ddot{a}}{a} \frac{1}{H^{2}}
$$

The term $\ddot{a} / a$ can be calculated from the second Friedmann equation, that for a matterdominated universe with bulk viscosity reads:

$$
\frac{\ddot{a}}{a}=-\frac{4 \pi G}{3}\left(\rho_{\mathrm{m}}-9 \zeta H\right)
$$


From the definition of $\tilde{\zeta}$ (see expression in $(12)$ ) we have that

$$
\zeta=\left(\frac{H_{0}}{24 \pi G}\right) \tilde{\zeta}
$$

On the other hand, from the first Friedmann equation (7) we have

$$
\rho_{\mathrm{m}}=\frac{3}{8 \pi G} H^{2}(a)
$$

So, substituting the expressions (34) and (35) in (33) we obtain:

$$
\frac{\ddot{a}}{a}=\frac{1}{2}\left(\tilde{\zeta} H_{0}-H(a)\right) H(a)
$$

Thus, substituting (36) in (32) yields

$$
q(a)=\frac{1}{2}\left(1-\tilde{\zeta} \frac{H_{0}}{H(a)}\right)
$$

Using equation (14) for $H(a)$ we arrive to

$$
q(a, \tilde{\zeta})=\frac{1}{2}\left[\frac{3-\tilde{\zeta}\left(1+2 a^{3 / 2}\right)}{3-\tilde{\zeta}\left(1-a^{3 / 2}\right)}\right]
$$

Figure 7 shows the behavior of expression (37). Note that this expression does not depend on $H_{0}$. We summarize the behavior of the deceleration parameter according to different values of $\tilde{\zeta}$ as follows:

- When $\tilde{\zeta}=0$ we have $q=\frac{1}{2}$ that corresponds to a matter-dominated universe with null bulk viscosity.

- When $\tilde{\zeta}=3$ we have $q=-1$ that corresponds to de Sitter universe.

- Case $0<\tilde{\zeta}<3$ : It is always a decreasing function from $q(0)=\frac{1}{2}$ to $q(\infty)=-1$ with a transition from positive to negative values in the value of the scale factor $a_{\mathrm{t}} \equiv[(3-\tilde{\zeta}) / 2 \tilde{\zeta}]^{2 / 3}$ (see expression $\left.(28)\right)$.

- Case $\tilde{\zeta}>3$ : When $a \rightarrow a^{*}$ then $q \rightarrow-\infty$ where $a^{*}=(1-3 / \tilde{\zeta})^{2 / 3}$ is the minimum value of the scale factor (see expression (31)) and when $a \rightarrow \infty$ then $q \rightarrow-1$. It is a negative and increasing function but it never becomes positive, its maximum value is -1 .

- Case $\tilde{\zeta}<0$ : When $a=0$ then $q=\frac{1}{2}$ and when $a \rightarrow a^{*}$ then $q \rightarrow \infty$ where $a^{*}=(1-3 / \tilde{\zeta})^{2 / 3}$ is the maximum value of the scale factor. It is a positive and increasing monotonic function where its minimum value is $\frac{1}{2}$.

From the expression (37) we obtain the deceleration parameter evaluated today as

$$
q(a=1, \tilde{\zeta})=\frac{1-\tilde{\zeta}}{2}
$$

Note that when $\tilde{\zeta}=1$ the transition from the decelerated to accelerated epochs of the universe takes place today. For $\tilde{\zeta}<1$ we have a decelerated universe in the present and for $\tilde{\zeta}>1$ we have an accelerated one today.

Assuming the best estimated values for $\tilde{\zeta}$ from table 1 , the deceleration parameter today is (see expression (38)): $q_{\text {today }}=-0.46 \pm 0.04$. 


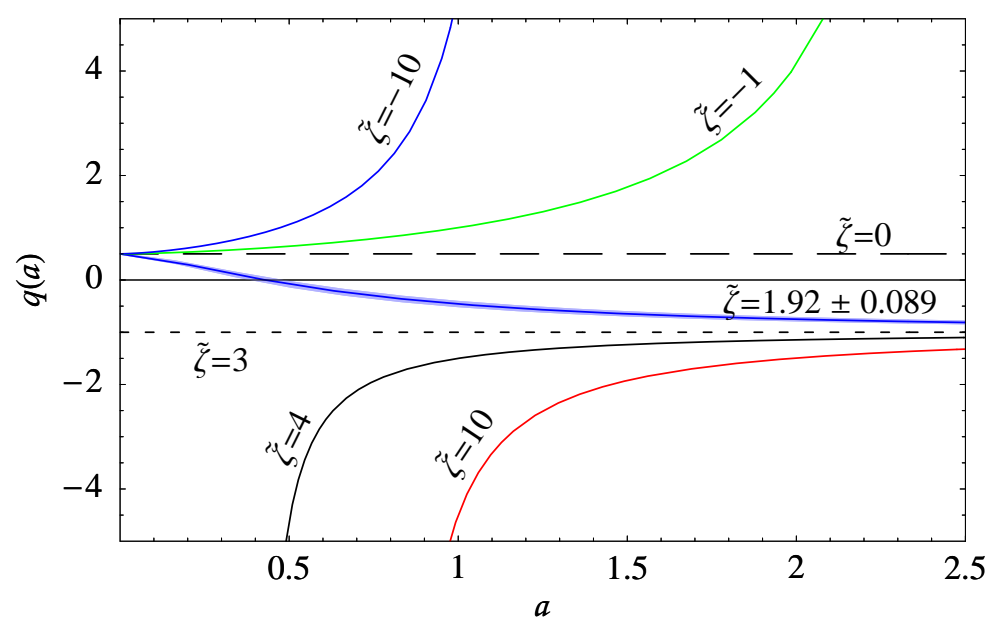

Figure 7. Plot of deceleration parameter $q(a, \tilde{\zeta})$ for different values of $\tilde{\zeta}$ (see expression (37)). The long dashed line corresponds to $\tilde{\zeta}=0$ (a flat matter-dominated universe with null bulk viscosity). The short dashed line corresponds to $\tilde{\zeta}=3$ (the de Sitter universe). The blue line with the blue band correspond to a model with $\tilde{\zeta}=1.922 \pm 0.089$. This is the best estimated value of $\tilde{\zeta}$ coming from the SCP "Union" $\mathrm{SNe}$ Ia data set analysis (see section 7 ). The band corresponds to the error at the $68.3 \%$ confidence level.

\subsection{The curvature scalar $R$.}

We calculate the curvature scalar $R$ in order to study the singularities of the model. For a flat universe it is defined as

$$
R=6\left[\frac{\ddot{a}}{a}+H^{2}\right]
$$

Using the expression (36) in (39) we obtain

$$
R=3\left[\tilde{\zeta} H_{0}+3 H(a)\right] H(a)
$$

Substituting the expression (14) for $H(a)$ into (40) yields

$$
R(a, \tilde{\zeta})=\frac{H_{0}^{2}}{3}\left[\frac{(3-\tilde{\zeta})^{2}}{a^{3}}+\frac{5 \tilde{\zeta}(3-\tilde{\zeta})}{a^{3 / 2}}+4 \tilde{\zeta}^{2}\right]
$$

Figure 8 shows some plots of expression (41). From this expression we note that:

- When $\tilde{\zeta}=0$ then $R=3 H_{0}^{2} / a^{3}$ that corresponds to a matter-dominated universe with null bulk viscosity.

- When $\tilde{\zeta}=3$ then $R=12 H_{0}^{2}$ that corresponds to de Sitter universe.

- Case $0<\tilde{\zeta}<3$ : When $a \rightarrow 0$ then $R \rightarrow \infty$ (the Big-Bang singularity). It is always a positive and decreasing function until to reach its minimum value $R=\frac{4}{3} H_{0}^{2} \tilde{\zeta}$ when $a \rightarrow \infty$.

- Case $\tilde{\zeta}>3$ : It is always a positive and increasing function from zero at $a=a^{*}$ (where $a^{*}=(1-3 / \tilde{\zeta})^{2 / 3}$ is the minimum value of the scale factor) until to reach its maximum value $R=\frac{4}{3} H_{0}^{2} \tilde{\zeta}$ when $a \rightarrow \infty$. 


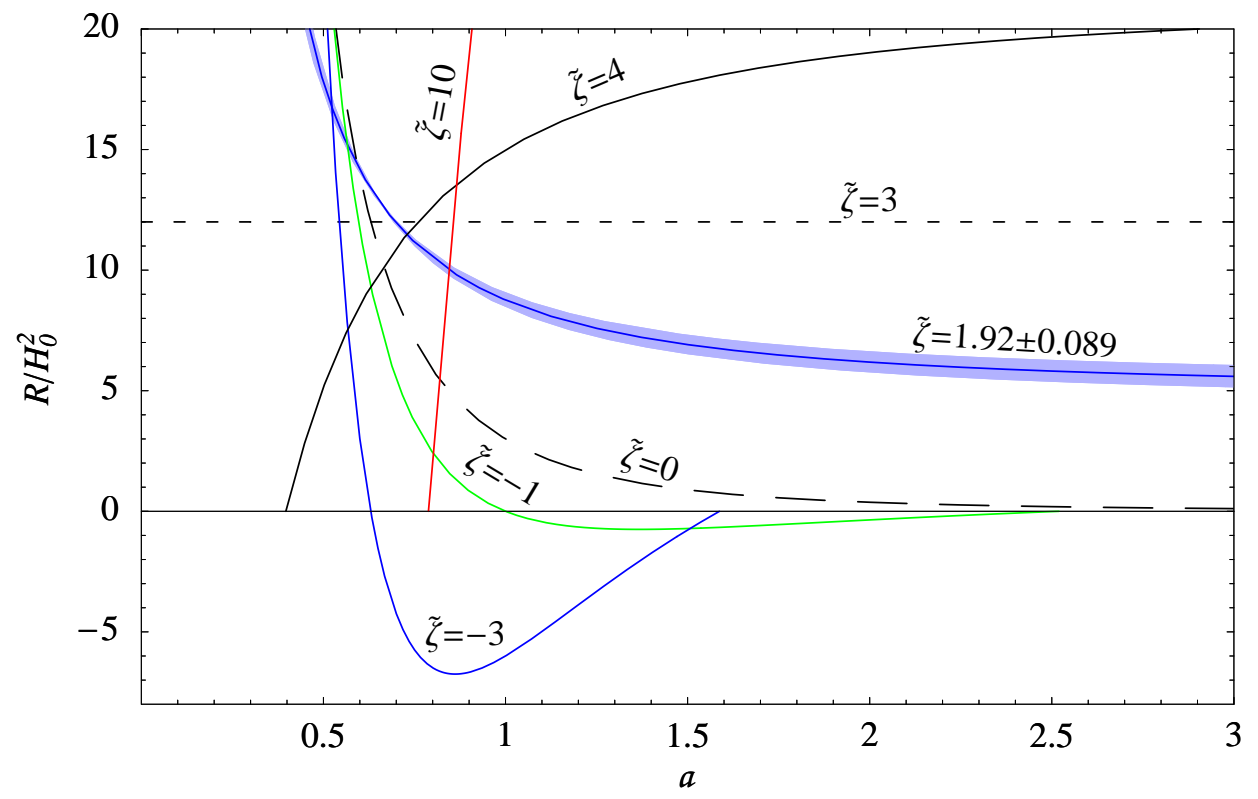

Figure 8. Plot of the curvature scalar $R(a, \tilde{\zeta})$ as a function of the scale factor for different values of $\tilde{\zeta}$ (see expression (41)). The long dashed line corresponds to $\tilde{\zeta}=0$ (a flat matter-dominated universe with null bulk viscosity). The short dashed line corresponds to $\tilde{\zeta}=3$ (the de Sitter universe). The blue line with the blue band correspond to a model with $\tilde{\zeta}=1.922 \pm 0.089$. This is the best estimated value of $\tilde{\zeta}$ coming from the SCP "Union" SNe Ia data set analysis (see section 7). The band corresponds to the error at the $68.3 \%$ confidence level.

- Case $\tilde{\zeta}<0$ : When $a \rightarrow 0$ then $R \rightarrow \infty$ (the Big-Bang singularity). The curvature scalar is zero at the values of the scale factor $a_{0}$ and $a^{*}$, where

$$
a_{0}=\left(\frac{\tilde{\zeta}-3}{4 \tilde{\zeta}}\right)^{2 / 3}
$$

and $a^{*}$ is the maximum value for the scale factor defined in expression (31). At $a_{0}$ there is a transition from positive to negative values of $R$. When $0<a<a_{0}$ the curvature scalar is a positive and decreasing function. For $a_{0}<a<a^{*}$ the value of $R$ is negative. The minimum value of the curvature scalar is $R=-\frac{3}{4} H_{0}^{2} \tilde{\zeta}^{2}$ at the value of the scale factor $\tilde{a}$

$$
\tilde{a}=\left[\frac{2}{5}\left(\frac{\tilde{\zeta}-3}{\tilde{\zeta}}\right)\right]^{2 / 3}
$$

\subsection{The matter density $\rho_{\mathrm{m}}$.}

We calculate the matter density $\rho_{\mathrm{m}}$ predicted for this model. We substitute the Hubble parameter $H(a)$ (expression (14)) into the expression (35) for the matter density, yielding

$$
\rho_{\mathrm{m}}(a, \tilde{\zeta})=\frac{H_{0}^{2}}{24 \pi G}\left[\tilde{\zeta}+\frac{3-\tilde{\zeta}}{a^{3 / 2}}\right]^{2}
$$




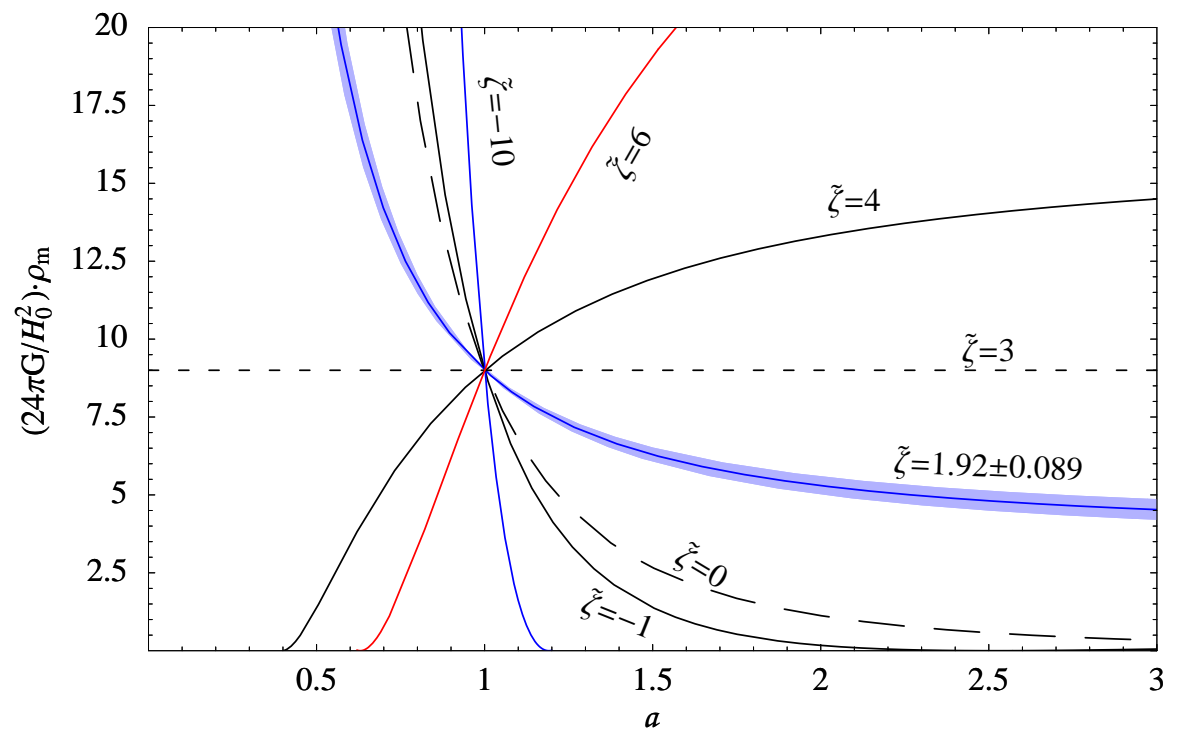

Figure 9. Plot of the matter density $\rho_{\mathrm{m}}(a, \tilde{\zeta})$ as a function of the scale factor for different values of $\tilde{\zeta}$ (see expression (44)). The long dashed line corresponds to $\tilde{\zeta}=0$ (a flat matter-dominated universe with null bulk viscosity). The short dashed line corresponds to $\tilde{\zeta}=3$ (the de Sitter universe). The blue line with the blue band correspond to a model with $\tilde{\zeta}=1.922 \pm 0.089$. This is the best estimated value of $\tilde{\zeta}$ coming from the SCP "Union" SNe Ia data set analysis (see section 7). The band corresponds to the error at the $68.3 \%$ confidence level.

Figure 9 shows some plots of expression (44). From this expression we note that:

- When $\tilde{\zeta}=0$ then $\rho_{\mathrm{m}}=\left(3 H_{0}^{2} / 8 \pi G\right) a^{-3}$ that corresponds to a matter-dominated universe with null bulk viscosity.

- When $\tilde{\zeta}=3$ then $\rho_{\mathrm{m}}=3 H_{0}^{2} / 8 \pi G$ that corresponds to de Sitter universe.

- Case $0<\tilde{\zeta}<3$ : When $a \rightarrow 0$ then $\rho_{\mathrm{m}} \rightarrow \infty$ (the Big-Bang singularity). It is a decreasing function until to reach its minimum value $\rho_{\mathrm{m}}=\left(H_{0}^{2} / 24 \pi G\right) \tilde{\zeta}^{2}$ when $a \rightarrow \infty$.

- Case $\tilde{\zeta}>3$ : When $a=a^{*}$ then $\rho_{\mathrm{m}}\left(a^{*}\right)=0$ where $a^{*}=(1-3 / \tilde{\zeta})^{2 / 3}$ is the minimum value of the scale factor (see expression (31) and section 4.2.3). It is an increasing monotonic function from zero until to reach its maximum value of $\rho_{\mathrm{m}}=\left(H_{0}^{2} / 24 \pi G\right) \tilde{\zeta}^{2}$ when $a \rightarrow \infty$. In this case, the matter density increases as in the Hoyle's steady state cosmology [84].

- Case $\tilde{\zeta}<0$ : When $a \rightarrow 0$ then $\rho_{\mathrm{m}} \rightarrow \infty$ (the Big-Bang singularity). It is a decreasing function until to reach its minimum value in $\rho_{\mathrm{m}}\left(a^{*}\right)=0$. 


\subsection{The age of the universe.}

We define the age of the universe as the elapsed time between the Big-Bang time $t_{\mathrm{B}}$ until today $t_{0}$. So, from the expression (19) for the case $\tilde{\zeta}=0$ we have

$$
\text { Age } \equiv\left|t_{\mathrm{B}}-t_{0}\right|=\frac{2}{3 H_{0}}, \quad \text { for } \quad \tilde{\zeta}=0
$$

And from the expression (26) for the case $\tilde{\zeta}<3$

$$
\text { Age } \equiv\left|t_{\mathrm{B}}-t_{0}\right|=-\frac{2}{\tilde{\zeta} H_{0}} \ln \left(1-\frac{\tilde{\zeta}}{3}\right), \quad \text { for } \quad \tilde{\zeta}<3
$$

Note that for the case $\tilde{\zeta} \geq 3$ the age of the universe is not defined. On the other hand, evaluating the expression (46) in the best estimated values of $\tilde{\zeta}$ and $H_{0}$ from the SCP "Union" SNe Ia data set (see table 1) yields an age of $14.957 \pm 0.422$ Gyr. The errors are at $68.3 \%$ confidence level. We found that this age is in perfect agreement with the constraints on the age of the universe coming from the oldest globular clusters [85]. Figure 10 shows the age of the universe for different values of $\tilde{\zeta}$ and the best estimate, the vertical lines correspond to $H_{0}=[55,75](\mathrm{km} / \mathrm{s}) \mathrm{Mpc}^{-1} \S$, it is the permitted region according to values of $H_{0}$ consistent with the distance moduli used to derive ages for Galactic globular clusters from the Hipparcos parallaxes. As a reference, the predicted age of the universe for $\Lambda$ CDM (Lambda Cold Dark Matter) cosmological model with a flat universe and using the best estimated values of $\Omega_{\mathrm{m} 0}$ and $H_{0}$ shown in table 1 is $13.750 \pm 0.29$ Gyr.

\section{Thermodynamics and the local entropy.}

The law of generation of the local entropy in the FRW space-time is found to be $[70,71,72]$

$$
T \nabla_{\nu} s^{\nu}=\zeta \nabla_{\nu} u^{\nu}=3 H \zeta
$$

where $T$ is the temperature and $\nabla_{\nu} s^{\nu}$ is the rate at which entropy is being generated in an unit volume. Then, the second law of the thermodynamics can be written as

$$
T \nabla_{\nu} s^{\nu} \geq 0
$$

which, from the expression (47), it implies that $3 H \zeta \geq 0$.

Since the Hubble parameter $H$ is positive in an expanding universe then $\zeta$ has to be positive in order to preserve the validity of the second law of the thermodynamics. Thus, equation (48) can be written for this model as

$$
\tilde{\zeta} \geq 0
$$

$\S$ Hereinafter the units of $H_{0}$ are expressed in $(\mathrm{km} / \mathrm{s}) \mathrm{Mpc}^{-1}$ 


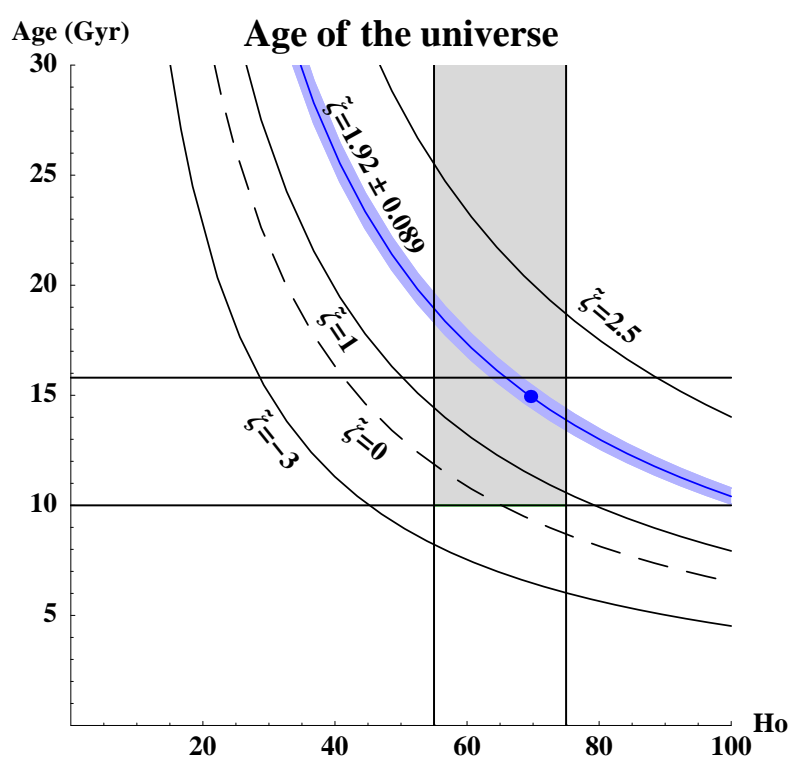

Figure 10. Plot of the age of the universe in units of Gigayears (Gyr) with respect to $H_{0}$ in units of $(\mathrm{km} / \mathrm{s}) \mathrm{Mpc}^{-1}$, for different values of $\tilde{\zeta}$ (see expression (45), (46)). The blue point is located at $14.957 \pm 0.422$ Gyr that corresponds to the best estimated value for the age of the universe coming from the SCP "Union" SNe Ia data set (where $H_{0}=69.62 \pm 0.59(\mathrm{~km} / \mathrm{s}) \mathrm{Mpc}^{-1}$, see section 7$)$. The blue line with the blue band correspond to a model evaluated at the best estimated value for $\tilde{\zeta}=1.922 \pm 0.089$. The vertical lines correspond to the interval $H_{0}=[55,75](\mathrm{km} / \mathrm{s}) \mathrm{Mpc}^{-1}$, it is the permitted region according to values of $H_{0}$ consistent with the distance moduli used to derive ages for Galactic globular clusters from the Hipparcos parallaxes (see ref. [85]). The horizontal lines corresponds to the constraint for the age of the universe from the oldest globular clusters (Age $=12.9 \pm 2.9 \mathrm{Gyr}[85]$ ). So, the shaded area is the consistent region for the age of the universe. The dashed line corresponds to $\tilde{\zeta}=0$, a flat matter-dominated universe with null bulk viscosity.

\section{Type Ia Supernovae test.}

We analyze and constrain the viability of the model using the type Ia supernovae (SNe Ia) observations and the entropy tests (see expression (49) and section 6). For that, we calculate the best estimated values for the parameters $\tilde{\zeta}$ and $H_{0}$ and the goodnessof-fit of the model to the data by $\chi^{2}$-minimization and then compute the confidence intervals for $\left(\tilde{\zeta}, H_{0}\right)$ and the probability density functions for $\tilde{\zeta}$ (marginalizing over $H_{0}$ ) to constrain their possible values.

We perform the statistical analysis using the "Union" SNe Ia data set of "The Supernova Cosmology Project" (SCP) [73] composed by 307 type Ia supernovae brought together from 13 independent data sets.

We use the definition of luminosity distance $d_{L}[3,5],[86]-[88]$ in a flat cosmology, it is

$$
d_{L}\left(z, \tilde{\zeta}, H_{0}\right)=c(1+z) \int_{0}^{z} \frac{d z^{\prime}}{H\left(z^{\prime}, \tilde{\zeta}, H_{0}\right)}
$$


where $H\left(z, \tilde{\zeta}, H_{0}\right)$ is the Hubble parameter (expression (13)) and ' $c$ ' is the speed of light. The theoretical distance moduli for the $k$-th supernova with redshift $z_{k}$ is defined as

$$
\mu^{\mathrm{t}}\left(z_{k}, \tilde{\zeta}, H_{0}\right) \equiv m-M=5 \log _{10}\left[\frac{d_{L}\left(z_{k}, \tilde{\zeta}, H_{0}\right)}{\mathrm{Mpc}}\right]+25
$$

where $m$ and $M$ are the apparent and absolute magnitudes of the SNe Ia respectively and the superscript ' $t$ ' stands for theoretical. We construct the statistical $\chi^{2}$ function as

$$
\chi^{2}\left(\tilde{\zeta}, H_{0}\right) \equiv \sum_{k=1}^{n} \frac{\left[\mu^{\mathrm{t}}\left(z_{k}, \tilde{\zeta}, H_{0}\right)-\mu_{k}\right]^{2}}{\sigma_{k}^{2}}
$$

where $\mu_{k}$ is the observational distance moduli for the $k$-th supernova, $\sigma_{k}^{2}$ is the variance of the measurement and $n$ is the amount of supernovae in the data set.

Once constructed the $\chi^{2}$ function (52), we numerically minimize it to compute the

"best estimates" for the free parameters of the model (i.e., $\tilde{\zeta}, H_{0}$ ). The $\chi^{2}$ function measures the goodness-of-fit of the model to data. The probability density function (pdf) is defined as

$$
\operatorname{pdf}\left(\tilde{\zeta}, H_{0}\right)=c t e \cdot \mathrm{e}^{-\chi^{2} / 2}
$$

where 'cte' is a normalization constant.

So, we minimize the $\chi^{2}$ function to obtain the results shown in table 1 . The confidence intervals for $\tilde{\zeta}$ and $H_{0}$ are shown in figure 11 . Note that at least at the $99.73 \%$ confidence level the possible values for $\tilde{\zeta}$ are inside of the interval $1<\tilde{\zeta}<3$. The models in this interval indicate the existence of a Big-Bang in the past of the universe, they have an accelerated expansion today and predict an age of the universe $\sim 13-18$ Gyr at the $99.73 \%$ confidence level (see figure 11 and subsections 4.2.1, 5.4).

In order to compare the results shown in table 1 (in particular the measurement of the goodness-of-fit to data $\chi_{\text {d.o.f. }}^{2}$ and the best estimated value for $H_{0}$ ) with other models we compute also the best estimated values for the $\Lambda$ CDM model using the same SNe Ia data set. For the $\Lambda$ CDM model, we assume a flat cosmology with $H_{0}$ and $\Omega_{\mathrm{m} 0}$ as free parameters (implying that the cosmological constant density parameter is $\left.\Omega_{\Lambda 0}=1-\Omega_{\mathrm{m} 0}\right)$. The results are shown in Table 1 .

We find that the best estimated values for $H_{0}$ and the goodness-of-fit to data $\chi_{\text {d.o.f. }}^{2}$ from $\Lambda$ CDM model are very similar to those obtained from the bulk viscous model using the same SCP "Union" SNe Ia data set and in agreement with that reported by 5 year WMAP $\left(H_{0}=70.5 \pm 1.3(\mathrm{~km} / \mathrm{s}) \mathrm{Mpc}^{-1}\right.$, see [83]).

\section{Marginalization over $H_{0}$}

Now, we construct a pdf for the bulk viscous coefficient $\tilde{\zeta}$ marginalizing over the Hubble constant $H_{0}$ in order to have to $\tilde{\zeta}$ as the only free parameter of the model. We use three different priors to marginalize $H_{0}$ :

(i) Constant prior over $H_{0}$ (see Appendix A). 


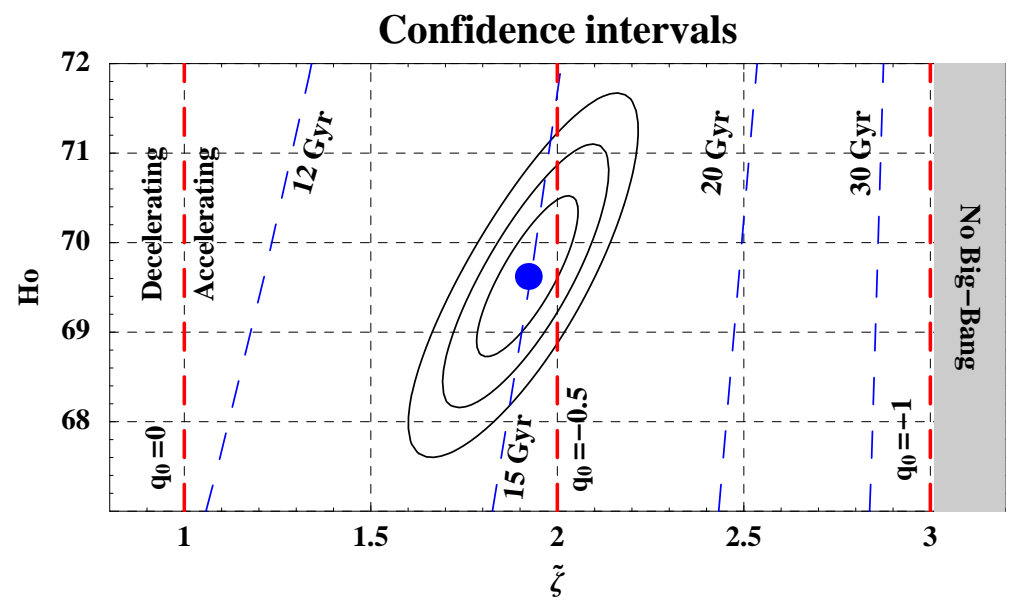

Figure 11. Confidence intervals for $\tilde{\zeta}$ and $H_{0}$ of a bulk viscous matter-dominated universe model with constant bulk viscosity. The best estimated values and confidence intervals were computed using the SCP "Union" SNe Ia data set where it is found $\tilde{\zeta}=1.922 \pm 0.089$ and $H_{0}=69.62 \pm 0.59(\mathrm{~km} / \mathrm{s}) \mathrm{Mpc}^{-1}$ as best estimates (see table 1$)$. The shown confidence intervals correspond to $68.3 \%, 95.4 \%$ and $99.73 \%$ of probability for the estimated value. $H_{0}$ is in units of $(\mathrm{km} / \mathrm{s}) \mathrm{Mpc}^{-1}$ and $\tilde{\zeta}$ is dimensionless. Note that at least at the $99.73 \%$ confidence level the possible values for $\tilde{\zeta}$ are inside of the interval $1<\tilde{\zeta}<3$ indicating an accelerating universe today with high confidence level (see subsection 4.2.1). The blue dashed lines show different ages of the universe according to the values of $\tilde{\zeta}$ and $H_{0}$ (see expression (46)). The red vertical dashed lines indicate values of the deceleration parameter evaluated today (see expression (38)) being the line $q_{0}=0$ the limit between a decelerated-accelerated universe today. The estimated age of the universe is $14.957 \pm 0.422 \mathrm{Gyr}$, where the error is at $68.3 \%$ confidence level. We found that this age is in perfect agreement with the constraints on the age of the universe coming from the oldest globular clusters [85] (see figure 10). The models that are in the grey region $(\tilde{\zeta} \geq 3)$ do not have a Big-Bang in the past of the universe as the label indicates.

(ii) Gaussian prior over $H_{0}$ centered at $H_{0}=70.5 \pm 1.3(\mathrm{~km} / \mathrm{s}) \mathrm{Mpc}^{-1}$ and $H_{0}=$ $72 \pm 8(\mathrm{~km} / \mathrm{s}) \mathrm{Mpc}^{-1}$ as reported by 5 year WMAP data [83] and HST Cepheid variable star observations [89] respectively (see Appendix B).

(iii) Dirac delta prior over $H_{0}$ centered at $H_{0}=70.5(\mathrm{~km} / \mathrm{s}) \mathrm{Mpc}^{-1}$ and $H_{0}=$ $72(\mathrm{~km} / \mathrm{s}) \mathrm{Mpc}^{-1}$ reported as mentioned above (see Appendix C).

\subsection{Marginalization assuming a constant prior over $H_{0}$.}

We marginalize over $H_{0}$ by assuming a constant prior as described in Appendix A. We use the $\chi_{\mathrm{cp}}^{2}$ function (A.18) instead of $\chi^{2}$ function (52) to perform the supernova analysis. The results are:

$$
\text { - } \tilde{\zeta}=1.922 \pm 0.089 \text {, with a } \chi_{\min }^{2}=331.30\left(\chi_{\text {d.o.f. }}^{2}=1.08\right) \text {. }
$$

The error is at $68.3 \%$ confidence level. We plot the pdf of $\tilde{\zeta}$ to show the value that maximizes such a function, i.e., the best estimated value of $\tilde{\zeta}$. This result is shown in 
Viscous model $\zeta=$ constant

\begin{tabular}{c|ccc|cc}
\hline Model & $H_{0}$ & $\tilde{\zeta}$ & $\Omega_{\mathrm{m} 0}$ & $\chi_{\min }^{2}$ & $\chi_{\text {d.o.f. }}^{2}$ \\
\hline Constant bulk viscosity & $69.62 \pm 0.59$ & $1.922 \pm 0.089$ & 1 & 314.57 & 1.031 \\
$\Lambda$ CDM & $70.01 \pm 0.59$ & - & $0.278 \pm 0.027$ & 311.84 & 1.022 \\
\hline
\end{tabular}

Table 1. Summary of the best estimates of $\tilde{\zeta}$ and $H_{0}$ for the bulk viscous matterdominated universe model with a constant bulk viscosity coefficient. Also, it is shown the best estimates for the $\Lambda \mathrm{CDM}$ model in order to compare the values of $H_{0}$ and $\chi_{\min }^{2}$ of both models. It is found that the best estimates for $H_{0}$ and their corresponding $\chi_{\min }^{2}$ computed for both models and using the same SNe Ia data set are almost the same value and in agreement with the reported value by 5 year WMAP $\left(H_{0}=70.5 \pm 1.3\right.$ $(\mathrm{km} / \mathrm{s}) \mathrm{Mpc}^{-1}$, see [83]). For the $\Lambda \mathrm{CDM}$ model, we assumed a flat cosmology with $H_{0}$ and $\Omega_{\mathrm{m} 0}$ as free parameters, where $\Omega_{\mathrm{m}}$ is the matter density parameter. Note that in the constant bulk viscous model the value of $\Omega_{\mathrm{m}}$ is not estimated but it is assumed to have the value of one as part of the model. The best estimates were computed by a Bayesian statistical analysis using the SCP "Union" 2008 compilation data set composed by $307 \mathrm{SNe}$ Ia from 13 independent data sets [73]. $H_{0}$ is in units of $(\mathrm{km} / \mathrm{s}) \mathrm{Mpc}^{-1}$ and $\tilde{\zeta}, \Omega_{\mathrm{m}}$ are dimensionless. The subscript "d.o.f." stands for degrees of freedom and the errors are at $68.3 \%$ confidence level. Figure 11 shows the confidence intervals.

\begin{tabular}{|c|c|c|c|}
\hline \multicolumn{4}{|c|}{$\begin{array}{c}\text { Viscous model } \zeta=\text { constant } \\
\text { Best estimates for } \tilde{\zeta}\end{array}$} \\
\hline Marg. & $\tilde{\zeta}$ & $\chi_{\min }^{2}$ & $\chi_{\text {d.o.f. }}^{2}$ \\
\hline (i) & $1.922 \pm 0.089$ & 331.30 & 1.08 \\
\hline (ii) & $1.941 \pm 0.084$ & 315.26 & 1.03 \\
\hline (iii) & $1.924 \pm 0.089$ & 314.91 & 1.02 \\
\hline (iv) & $2.026 \pm 0.051$ & 316.75 & 1.03 \\
\hline$(\mathrm{v})$ & $2.195 \pm 0.049$ & 330.41 & 1.07 \\
\hline
\end{tabular}

Table 2. Summary of the best estimates of $\tilde{\zeta}$ for the bulk viscous matter-dominated universe model with a constant bulk viscosity coefficient. The best estimates were computed by a Bayesian statistical analysis using the SCP "Union" 2008 compilation data set composed by $307 \mathrm{SNe}$ Ia from 13 independent data sets [73]. The errors are at $68.3 \%$ confidence level and $\tilde{\zeta}$ is dimensionless. Figure 12 shows the probability density functions for $\tilde{\zeta}$. We have marginalized over the Hubble constant $H_{0}$ using three different priors described in section 8 . In the first column of the table the label "Marg." indicates the marginalization method over $H_{0}$ used to obtain the results. The numbers correspond to:

(i) Constant prior over $H_{0}$ (see Appendix A).

(ii) Gaussian prior over $H_{0}$ centered at $H_{0}=70.5 \pm 1.3(\mathrm{~km} / \mathrm{s}) \mathrm{Mpc}^{-1}$, as reported by 5 year WMAP data [83] (see Appendix B).

(iii) Gaussian prior over $H_{0}$ centered at $H_{0}=72 \pm 8(\mathrm{~km} / \mathrm{s}) \mathrm{Mpc}^{-1}$, as reported by HST Cepheid variable star observations [89] (see Appendix B).

(iv)Dirac delta prior over $H_{0}$ located at $H_{0}=70.5(\mathrm{~km} / \mathrm{s}) \mathrm{Mpc}^{-1}$, as reported by 5 year WMAP data (see Appendix C).

(v)Dirac delta prior over $H_{0}$ located at $H_{0}=72(\mathrm{~km} / \mathrm{s}) \mathrm{Mpc}^{-1}$, as reported by HST Cepheid variable star observations (see Appendix C). 
table 2 and figure 12.

\subsection{Marginalization assuming a Gaussian prior over $H_{0}$.}

We marginalize over $H_{0}$ by assuming a Gaussian prior as described in Appendix B. We use two different central values for the Gaussian prior for $H_{0}$, they are $H_{0}=70.5 \pm 1.3(\mathrm{~km} / \mathrm{s}) \mathrm{Mpc}^{-1}$ and $H_{0}=72 \pm 8(\mathrm{~km} / \mathrm{s}) \mathrm{Mpc}^{-1}$ as suggested by 5 year WMAP data [83] and HST Cepheid variable star observations [89] respectively. See section Appendix B for details. The results are shown in table 2 and figure 12.

\subsection{Marginalization assuming a Dirac delta prior over $H_{0}$.}

Finally, we marginalize over $H_{0}$ by assuming a Dirac delta prior as described in Appendix C. In practice, it means to assume a specific value for $H_{0}$. In the same way as above we use two different values for $H_{0}$, they are $H_{0}=70.5(\mathrm{~km} / \mathrm{s}) \mathrm{Mpc}^{-1}$ and $H_{0}=72(\mathrm{~km} / \mathrm{s}) \mathrm{Mpc}^{-1}$. The results are shown in table 2 and figure 12 .

\section{Conclusions.}

We performed a detailed study of a bulk viscous matter-dominated universe model with constant bulk viscous coefficient $\zeta$. The only component of this model is a pressureless fluid with an assumed constant bulk viscosity driving the present accelerated expansion of the universe. We analyzed the different possible scenarios for the behavior and evolution of the universe predicted by the model according to the value of the dimensionless bulk viscous coefficient $\tilde{\zeta}$. In general, the model predicts an expanding universe for any value of $\tilde{\zeta}$ and we find that for values of $\tilde{\zeta}$ in the range $0<\tilde{\zeta}<3$ the model predicts an universe with a Big-Bang in the past and then starting with a decelerated expansion epoch followed by a transition to an accelerated epoch in late times.

The case $\tilde{\zeta}=3$ corresponds to the de Sitter universe. When $\tilde{\zeta}>3$ the universe is always in an accelerated expansion and the value of the scale factor tends to infinity in the future (in a similar way than the de Sitter universe) and where the curvature scalar goes to a positive constant value. In this case there is not a Big-Bang nor a defined age nor origin of the universe because the scale factor has a minimum value greater than zero in the past of the universe (when $t \rightarrow-\infty$ ), where also, the curvature scalar and the matter density are zero, i.e., we have the Einstein static universe in the past.

When $\tilde{\zeta}<0$ the universe is always in a decelerated expansion and the scale factor tends to a finite value in the future (it tends to the Einstein static universe in the future) where also, the curvature scalar and the matter density go to zero. For $\tilde{\zeta}$ in this range there is always a Big-Bang in the past.

For any value of $\tilde{\zeta}$ there does not exist any recollapse epoch or transition between expansion to contraction epochs. When $\tilde{\zeta}=1$ the transition between a decelerated to accelerated epoch takes place today (i.e., $q_{0}=0$ for $\tilde{\zeta}=1$ ). For $0<\tilde{\zeta}<1$ the transition 
Probability density functions
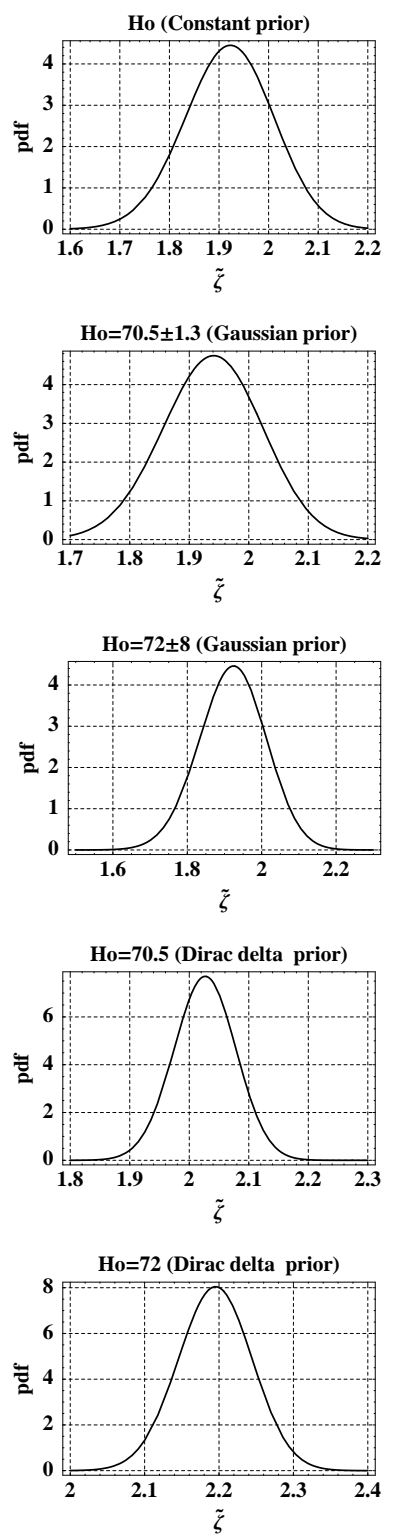

Figure 12. Probability distribution functions for the coefficient $\tilde{\zeta}$ in a bulk viscous matter-dominated universe model with a constant bulk viscosity. We have marginalized over the Hubble constant $H_{0}$ using three different priors described in section 8. The best estimates and pdf's were computed by a Bayesian statistical analysis using the SCP "Union" 2008 compilation data set composed by 307 SNe Ia from 13 independent data sets [73]. Table 2 summarizes the best estimated values of $\tilde{\zeta}$ using the different marginalizations. The first figure (on top) corresponds to the marginalization using a constant prior over $H_{0}$ (see Appendix A). The second and third figures (from top to bottom) correspond to the marginalization using a Gaussian prior over $H_{0}$ centered at $H_{0}=70.5 \pm 1.3(\mathrm{~km} / \mathrm{s}) \mathrm{Mpc}^{-1}$ and $H_{0}=72 \pm 8(\mathrm{~km} / \mathrm{s}) \mathrm{Mpc}^{-1}$ as reported by 5 year WMAP data [83] and HST Cepheid variable star observations [89] respectively. The fourth and fifth figures correspond to the marginalization using a Dirac delta prior over $H_{0}$ located at $H_{0}=70.5(\mathrm{~km} / \mathrm{s}) \mathrm{Mpc}^{-1}$ and $H_{0}=72(\mathrm{~km} / \mathrm{s}) \mathrm{Mpc}^{-1}$, as mentioned above. 
between the deceleration-acceleration epoch takes place in the future and for $1<\tilde{\zeta}<3$ the transition took place in the past.

On the other hand, we estimate the values of $\tilde{\zeta}$ and $H_{0}$ using the SCP Union SNe Ia data set where it is found that the estimated values of $\tilde{\zeta}$ are positives, in agreement with the second law of thermodynamics. We note that the best estimated value for $H_{0}$ is in agreement with that reported by 5 year WMAP and estimated from $\Lambda$ CDM model (using the same SNe Ia data set) and the minimum value of the $\chi^{2}$ function $\left(\chi_{\text {d.o.f. }}^{2}\right)$ obtained is of the same order than that obtained for $\Lambda$ CDM model indicating that this is a competitive model to fit the supernova observations.

We estimate and compare the age of the universe predicted by this model with the constraints on the age coming from the oldest globular cluster observations. We find that the best estimated values for the age of the universe is $14.95 \pm 0.42$ Gyr that is in agreement with the constraint in the age coming from the oldest globular clusters.

The present model has been constrained using SNe Ia data. It means that we have constrained the model using information (observations) of the past of the universe until a redshift $z \lesssim 1.5$. So, SNe Ia observations are not enough to trace the whole evolution of the universe from the Big-Bang until the present time. Therefore, it would be important to constrain the model using also cosmological observations that can provide information of the early universe like the observations of the CMB anisotropies from the WMAP experiment and the large scale structure (LSS) from SDSS experiment. A preliminary work in that sense has been already done in [56] showing (but not in a conclusive way) that when the model is constrained using the shift parameter $R$ of CMB and the parameter $A$ measuring the BAO peak from SDSS, in addition to the Gold 2006 SNe Ia data set, the estimated value of $\tilde{\zeta}$ is negative at $99.7 \%$ confidence level, violating the second law of thermodynamics and the estimated value of $H_{0}$ is lower $\left(H_{0} \sim 53(\mathrm{~km} / \mathrm{s}) \mathrm{Mpc}^{-1}\right)$ than that reported by 5 year WMAP and HST Cepheid variable star observations and giving a bad goodness-of-fit to data. Considering these facts we may conjecture that this model does not work well for early times of the universe and that in order to be a viable model the bulk viscosity should be triggered just until late times.

Another problem of the model is to explain the origin of the bulk viscosity from known or new physics. Some proposals in that sense have been explored, for instance in [49] and [50], where it is proposed a mechanism to generate the bulk viscosity by the decay of dark matter particles into relativistic products.

Finally, we explore also the sensitivity of the results to different ways of marginalization over $H_{0}$ when we estimate just the coefficient $\tilde{\zeta}$. We find that it is almost negligible the difference in the estimations of $\tilde{\zeta}$ when $H_{0}$ is marginalized assuming constant and Gaussian priors centered in the values reported by 5 year WMAP and HST Cepheid variable star observations. However, when $H_{0}$ is marginalized by assuming a specific value (a Dirac delta prior located at the values reported as mentioned above) the estimated values of $\tilde{\zeta}$ have a small increase and the values of $\chi_{\text {d.o.f. }}^{2}$ increase as well (i.e., we have a worse fit to data). 


\section{Acknowledgements.}

We thank Jose Antonio González for his comments on the constraints for the age of the universe, Francisco S. Guzmán and Olivier Sarbach for useful discussions during the preparation of this work. We also thank John Barrow for his useful comments on previous works done on this topic. We acknowledge to the Instituto Avanzado de Cosmología (IAC) for its partial support and useful seminars. This work was in part supported by grants SNI-20733, CIC-UMSNH No. 4.8, UMSNH-CA-22 and COECyTFIFOECYT 2008.

\section{Appendix A. Marginalization assuming a constant prior over $H_{0}$.}

To construct a pdf that depends only on the parameter $\tilde{\zeta}$ we use the process of marginalization over $H_{0}$ in order to eliminate the dependence of the pdf with respect to the parameter $H_{0}$.

The following procedure can be applied for any other model. It is, in a model with several parameters where it is necessary to reduce the number of free parameters or, for some reason, to eliminate the dependance of the model from some particular free parameters (for instance: $H_{0}$ ) in order to compute the best estimated values of the other free parameters that we keep (in our case: $\tilde{\zeta}$ ) the solution is to marginalize over the parameters that we want to eliminate.

In the present work we assume three different prior distribution functions for $H_{0}$ : constant, Gaussian and Dirac delta. In this appendix we describe the marginalization using a constant prior for $H_{0}$.

We start by multiplying and dividing by $H_{0}$ the equation (50)

$$
d_{L}\left(z, \tilde{\zeta}, H_{0}\right)=\frac{c(1+z)}{H_{0}} \int_{0}^{z} \frac{H_{0} d z^{\prime}}{H\left(z^{\prime}, \tilde{\zeta}, H_{0}\right)}
$$

Next, we define a new dimensionless luminosity distance as $D_{L}(z, \tilde{\zeta}) \equiv H_{0} \cdot d_{L}\left(z, \tilde{\zeta}, H_{0}\right) / c$. Then

$$
D_{L}(z, \tilde{\zeta})=c(1+z) \int_{0}^{z} \frac{d z^{\prime}}{E\left(z^{\prime}, \tilde{\zeta}\right)}
$$

where $D_{L}(z, \tilde{\zeta})$ does not depend on $H_{0}$ anymore and $E(z, \tilde{\zeta}) \equiv H\left(z, \tilde{\zeta}, H_{0}\right) / H_{0}$.

The theoretical distance moduli (51) becomes

$\mu^{\mathrm{t}}\left(z, \tilde{\zeta}, H_{0}\right)=5 \log _{10}\left(\frac{D_{L}(z, \tilde{\zeta}) \cdot c}{H_{0} \cdot \mathrm{Mpc}}\right)+25 \equiv 5 \log _{10}\left(\frac{D_{L}(z, \tilde{\zeta})}{\tilde{H}_{0}}\right)+25$

where we have defined a dimensionless "Hubble parameter" $\tilde{H}_{0} \equiv H_{0} \cdot \mathrm{Mpc} / c$. It is useful to define a new theoretical distance moduli that does not depend on $H_{0}$ anymore as

$$
\tilde{\mu}^{\mathrm{t}}(z, \tilde{\zeta}) \equiv 5 \log _{10}\left[D_{L}(z, \tilde{\zeta})\right]+25
$$


Then, the expression (A.3) for the distance moduli becomes

$$
\mu^{\mathrm{t}}\left(z, \tilde{\zeta}, H_{0}\right)=\tilde{\mu}^{\mathrm{t}}(z, \tilde{\zeta})-5 \log _{10}\left[\tilde{H}_{0}\right]
$$

Now we construct the $\chi^{2}$ function (52) with these new definitions as

$$
\chi^{2}\left(\tilde{\zeta}, H_{0}\right)=\sum_{i=1}^{n}\left(\frac{\tilde{\mu}^{\mathrm{t}}\left(z_{i}, \tilde{\zeta}\right)-\mu_{i}^{\mathrm{obs}}-5 \log _{10} \tilde{H}_{0}}{\sigma_{i}}\right)^{2}
$$

where $\mu_{i}^{\text {obs }}$ is the observed distance moduli and $\sigma_{i}$ its variance.

We rewrite the expression (A.6) as

$$
\begin{aligned}
\chi^{2}\left(\tilde{\zeta}, H_{0}\right)= & \sum_{i=1}^{n}\left(\frac{\tilde{\mu}_{i}^{\mathrm{t}}-\mu_{i}^{\mathrm{obs}}}{\sigma_{i}}\right)^{2}-2\left(5 \log _{10} \tilde{H}_{0}\right) \sum_{i=1}^{n}\left(\frac{\tilde{\mu}_{i}^{\mathrm{t}}-\mu_{i}^{\mathrm{obs}}}{\sigma_{i}^{2}}\right) \\
& +\left(5 \log _{10} \tilde{H}_{0}\right)^{2} \sum_{i=1}^{n}\left(\frac{1}{\sigma_{i}^{2}}\right)
\end{aligned}
$$

If we define \|

$$
A \equiv \sum_{i=1}^{n}\left(\frac{\tilde{\mu}_{i}^{\mathrm{t}}-\mu_{i}^{\mathrm{obs}}}{\sigma_{i}}\right)^{2}, \quad B \equiv \sum_{i=1}^{n} \frac{\tilde{\mu}_{i}^{\mathrm{t}}-\mu_{i}^{\mathrm{obs}}}{\sigma_{i}^{2}}, \quad C \equiv \sum_{i=1}^{n} \frac{1}{\sigma_{i}^{2}}
$$

then we can express (A.7) as

$$
\chi^{2}\left(\tilde{\zeta}, H_{0}\right)=A-2 B x+C x^{2}
$$

where

$$
x \equiv 5 \log _{10}\left(\tilde{H}_{0}\right) .
$$

Note that all the dependence of the $\chi^{2}$ function with respect to $H_{0}$ is now in the $x$ variable. Remind that the pdf is defined as

$$
\operatorname{pdf}\left(\tilde{\zeta}, \tilde{H}_{0}\right)=c t e \cdot \mathrm{e}^{-\chi^{2} / 2}
$$

where "cte" is a normalization constant. So, we marginalize the pdf (A.11) over $\tilde{H}_{0}$ computing the following integration

$$
\operatorname{pdf}(\tilde{\zeta})=\int_{-\infty}^{\infty} \operatorname{pdf}\left(\tilde{\zeta}, \tilde{H}_{0}\right) \cdot \operatorname{pdf}\left(\tilde{H}_{0}\right) d \tilde{H}_{0}
$$

where $\operatorname{pdf}\left(\tilde{H}_{0}\right)$ is the prior probability density function for $\tilde{H}_{0}$. In general, the integration (A.12) has to be done over the range of all the possible values of the parameter to be marginalized. In this case the range of possible values for $H_{0}$, or equivalently $\tilde{H}_{0}$, is $(-\infty, \infty)$.

If we take the case where the prior for $\tilde{H}_{0}$ is a constant then:

$$
\operatorname{pdf}(\tilde{\zeta})=c t e \int_{-\infty}^{\infty} \operatorname{pdf}\left(\tilde{\zeta}, \tilde{H}_{0}\right) d \tilde{H}_{0}
$$

\| Note that these expressions do not depend on $H_{0}$ anymore. 
A constant prior means that we do not prefer any particular value for $\tilde{H}_{0}$, i.e., any value for $\tilde{H}_{0}$ has the same probability of being. To solve the integral (A.13) we do a change of variable from $\tilde{H}_{0}$ to $x$ (see expression (A.10)). At the same time, we substitute the expression (A.9) into (A.11) and then (A.11) into (A.13), yielding

$\operatorname{pdf}(\tilde{\zeta})=\operatorname{cte}\left(\frac{\ln 10}{5}\right) \exp \left[\frac{1}{2}\left(\frac{\tilde{B}^{2}}{C}-A\right)\right] \int_{-\infty}^{\infty} \exp \left[-\frac{C}{2}\left(x-\frac{\tilde{B}}{C}\right)^{2}\right] d x$

where $\tilde{B} \equiv B+(\ln 10) / 5$.

We can see that the integral (A.14) has the form of a Gaussian distribution with value:

$$
1=\frac{1}{\sigma \sqrt{2 \pi}} \int_{-\infty}^{\infty} \exp \left[-\frac{(x-\bar{x})^{2}}{2 \sigma^{2}}\right] d x
$$

where $\bar{x}$ is the mean and $\sigma^{2}$ is the variance of the distribution.

Therefore the expression (A.14) becomes

$$
\operatorname{pdf}(\tilde{\zeta})=\operatorname{cte}\left(\frac{\ln 10}{5}\right) \sqrt{\frac{2 \pi}{C}} \exp \left[-\frac{1}{2}\left(A-\frac{\tilde{B}^{2}}{C}\right)\right]
$$

so that the $\operatorname{pdf}(\tilde{\zeta})$ does not depend on $H_{0}$ anymore. Note that it was not necessary any numerical integration of the expression (A.13). We can express this $\operatorname{pdf}(\tilde{\zeta})$ in terms of a new $\chi_{\mathrm{cp}}^{2}$ function like

$$
\operatorname{pdf}(\tilde{\zeta})=a \cdot \mathrm{e}^{-\chi_{\mathrm{cp}}^{2} / 2}
$$

where $a \equiv c t e \cdot \sqrt{2 \pi} \ln 10 /(5 \sqrt{C})$ and

$$
\chi_{\mathrm{cp}}^{2}(\tilde{\zeta}) \equiv A(\tilde{\zeta})-\frac{[B(\tilde{\zeta})+\ln (10) / 5]^{2}}{C}
$$

This new $\chi_{\mathrm{cp}}^{2}$ function does not depend on $H_{0}$ anymore. The label "cp" stands for constant prior for $H_{0}$.

\section{Appendix B. Marginalization assuming a Gaussian prior over $H_{0}$.}

We perform the marginalization assuming a Gaussian probability distribution function for $H_{0}$ centered at $H_{0}^{*}$ and with standard deviation $\sigma^{*}$. So, the pdf $\left(H_{0}\right)$ prior with the form of a Gaussian distribution for $H_{0}$ is

$$
\operatorname{pdf}\left(H_{0}\right)=\exp \left[-\frac{1}{2}\left(\frac{H_{0}-H_{0}^{*}}{\sigma^{*}}\right)^{2}\right]
$$

With this, the expression (A.12) becomes

$$
\operatorname{pdf}(\tilde{\zeta})=c t e \cdot \int_{-\infty}^{\infty} \mathrm{e}^{-\chi^{2} / 2} \exp \left[-\frac{1}{2}\left(\frac{H_{0}-H_{0}^{*}}{\sigma^{*}}\right)^{2}\right] d H_{0}
$$


where the $\chi^{2}$ function is given by (A.9) and "cte" is a normalization constant.

In the present work we use two different central values for the Gaussian prior coming from two different observations. One of them, is that coming from the 5 year WMAP observations where $H_{0}^{*}=70.5(\mathrm{~km} / \mathrm{s}) \mathrm{Mpc}^{-1}$ with an standard deviation of $\sigma^{*}=1.3(\mathrm{~km} / \mathrm{s}) \mathrm{Mpc}^{-1}$. The other central value is $H_{0}^{*}=72(\mathrm{~km} / \mathrm{s}) \mathrm{Mpc}^{-1}$ with an standard deviation of $\sigma^{*}=8(\mathrm{~km} / \mathrm{s}) \mathrm{Mpc}^{-1}$ as reported by the HST Cepheid variable star observations.

In practice we perform the numerical integration of the expression (B.2) in the interval $H_{0}=[55,85](\mathrm{km} / \mathrm{s}) \mathrm{Mpc}^{-1}$ considering that this is a suitable and representative interval that include almost the $100 \%$ of the probability density for $H_{0}$.

\section{Appendix C. Marginalization assuming a Dirac delta prior over $H_{0}$.}

It means to assume a specific value of $H_{0}$. It is like to think that the value of the Hubble constant is $H_{0}^{*}$ that has been measured with an infinite accuracy (with standard deviation equal to zero), so that its $\mathbf{p d f}\left(H_{0}\right)$ has the form of a Dirac delta function.

Clearly, this assumption is just an idealization to simplify the work and it does not correspond to the reality. However, for some cases it is a good approximation. This prior has the advantage of that once assumed it is very simple to perform the integration of the expression (A.12) using the Dirac delta properties. Thus, the prior with the form of a Dirac delta for $H_{0}$ is

$$
\operatorname{pdf}\left(H_{0}\right)=\delta\left(H_{0}-H_{0}^{*}\right)
$$

With this, the expression (A.12) becomes

$$
\operatorname{pdf}(\tilde{\zeta})=c t e \cdot \mathrm{e}^{-\chi^{2}\left(\tilde{\zeta}, H_{0}^{*}\right) / 2}
$$

where "cte" is a normalization constant.

\section{References.}

[1] Riess A G et al, 1998 Astron. J. 116 1009-1038

[2] Perlmutter S et al, 1999 Astrophys. J. 517565

[3] Riess A G et al, 2004 Astrophy. J. 607, 665-687

[4] Astier P et al, 2006 Astronomy and Astrophysics 447 31-48

[5] Riess A G et al, 2007 Astrophy. J. 659 98-121

[6] Davis et al. 2007 Astrophys. J. 666 716-725.

[7] Wood-Vasey et al, 2007 Astrophys. J. 666 694-715

[8] Bennett C L et al, 2003 Astrophys. J. 1481

[9] Tegmark M et al, 2004 Phys. Rev. D. 69103501

[10] Caldwell R R, Dave R and Steinhardt P J 1998 Appl. Space Sci. 261303

[11] Zlatev I, Wang L and Steinhardt P J 1999 Phys. Rev. Lett. 82896

[12] Wang L, Caldwell R R, Ostriker J P and Steinhardt P J 2000 Astrophys. J. 53017

[13] Steinhardt P J 2003 Phil. Trans. R. Soc. A 3612497

[14] Peebles P J E 2003 Rev. Mod. Phys. 75 559-606

[15] Weinberg S 1989 Rev. Mod. Phys. 611

[16] Carroll S M, Press W H and Turner E L 1992 Annu. Rev. Astron. Astrophys. 30 499-542 
[17] Carroll S M 2001 Living Reviews in Relativity 41

[18] Padmanabhan T 2003 Physics Reports 380, issues 5-6, 235-320

[19] Steinhardt P J 1997 Critical Problems in Physics, edited by Fitch V L and Marlow D R (Princeton University Press, Princeton, NJ)

[20] Zlatev I, Wang L and Steinhardt P J 1999 Phys. Rev. Lett. 82896

[21] Steinhardt P J, Wang L and Zlatev I 1999 Phys. Rev. D 59123504

[22] Heller M, Klimek Z and Suszycki L 1973 Astrophy. and Space Science 20 205-212

[23] Heller M and Klimek Z 1975 Astrophys. and Space Science 33 L37-L39

[24] Diosi L, Keszthelyi B, Lukács and Paál G 1984 Acta Phys. Pol. B 15 909; 1985 Phys. Lett. B 15723

[25] Morikawa M and Sasaki M 1985 Phys. Lett. B 16559

[26] Waga I, Falcao R C and Chanda R 1986 Phys. Rev. D 331839

[27] Barrow J 1986 Phys. Lett. B 180335

[28] Padmanabhan T and Chitre S M 1987 Phys. Lett. A 120443

[29] Gron O 1990 Astrophys. and Space Science 173 191G

[30] Beesham A 1993 Physical Rev. D 483539

[31] Diego Pavon and Winfried Zimdahl 1993 Phys. Lett. A 179 261-265

[32] Maartens R 1995 Class. Quantum Grav. 12 1455-1465

[33] Coley A A, van den Hoogen R J and Maartens R 1996 Phys. Rev. D 54 1393-1397

[34] Zimdahl W 1996 Physical Rev. D 535483

[35] Maartens R and Méndez V 1997 Physical Rev. D 551937

[36] Hiscock W A and Salmonson J 1991 Physical Rev. D 433249

[37] Kremer G M and Devecchi F P 2003 Physical Rev. D 67047301

[38] Fabris J C Goncalves S V B and de Sá Ribeiro R 2006 Gen. Relativ. Gravit. 38(3), 495-506

[39] Ming-Guang Hu and Xin-He Meng 2006 Phys. Letters B 635 186-194

[40] Jie Ren and Xin-He Meng 2006 Phys. Lett. B 633 1-8

[41] Jie Ren and Xin-He Meng 2006 Phys. Lett. B 636 5-12

[42] Marek Szydlowski and Orest Hrycyna 2007 Annals Phys 322 2745-2775

[43] Debnath P S, Paul B C and Beesham A 2007 Phys. Rev. D 76123505

[44] Colistete R Jr, Fabris J C, Tossa J and Zimdahl W 2007 Phys. Rev. D 76, 103516, 1-13

[45] Singh C P, Kumar S and Pradhan A 2007 Class. Quantum Grav. 24 455-474

[46] Meng X M and Dou X 2008 [arXiv:0812.4904]

[47] Zimdahl W 2000 Phys. Rev. D 61083511

[48] Zimdahl W, Schwarz D, Balakin A B and Pavón D 2001 Phys. Rev. D 64063501

[49] Wilson J R, Mathews G J and Fuller G M 2007 Physical Rev. D. 75043521

[50] Mathews G J, Lan N Q and Kolda C 2008 Phys. Rew. D 78, 043525

[51] Wang Y and Mukherjee P 2006 Astrophy. J. 6501

[52] Eisenstein D J et al, 2005 (SDSS Collaboration) Astrophy. J. 633, 560-574

[53] Klimek Z 1974 Acta Cosmologica 249

[54] Heller M and Suszycki L 1974 Acta Phys. Pol B5 345

[55] Klimek Z 1975 Acta Cosmologica 349

[56] Avelino A, Nucamendi U and Guzmán F S 2008 AIP Conf. Proc. 1026, 300-302. Proc. of the XI Mexican Workshop on Particles and Fields, Tuxtla Gutiérrez, México, nov 7-12, $200 \%$. [arXiv:0801.1686]

[57] John D Barrow 1987 Phys. Lett. B 180 335-339

[58] John D Barrow 1988 Nucl. Phys. B 310 743-763

[59] John D Barrow 1990 String-driven Inflation. In The formation and evolution of cosmic strings eds. G Gibbons \& T Vasasparti 449-464 CUP Cambridge.

[60] Avelino A, Nucamendi 2008 AIP Conf. Proc. 1083, 1-4. Proc. of the III International Meeting on Gravitation and Cosmology, Morelia, México, May 26-30, 2008. [arXiv:0810.0303]

[61] Nojiri S and Odintsov S D 2005 Phys. Rev. D 72:023003 
[62] Capozziello S et al, 2006 Phys. Rev. D 73:043512

[63] Mubasher Jamil and Muneer Ahmad Rashid 2008 Eur. Phys. J. C 56 429-434

[64] Hrvoje Stefancic 2008 [arXiv:0811.4548]

[65] Hrvoje Stefancic 2008 [arXiv:0812.3796]

[66] Maartens R 1996 in Proceedings of Hanno Rund Workshop on Relativity and Thermodynamics, South Africa, June 1996 (unpublished) [arXiv:9609119]

[67] Okumura H and Yonezawa F 2003 Physica A 321207

[68] Ilg P and Ottinger H C 1999 Phys. Rev. D 61023510

[69] Xinzhong C and Spiegel E A 2001 Mon. Not. R, Astron. Soc. 323865

[70] Weinberg S 1972 Gravitation and Cosmology: Principles and Applications of the General Theory of Relativity (John Wiley \& Sons, Inc., New York); 1971 Astrophys. J. 168, 175.

[71] Misner C W, Thorne K S and Wheeler J A 1973 Gravitation (W. H. Freeman and Company) p 567

[72] Hofmann S, Schwarz D J and Stoecker H 2001 Phys. Rev. D 64083507

[73] Kowalski M et al (The Supernova Cosmology Project) 2008 Astrophys. J. 686 749-778

[74] Eckart C 1940 Physical Rev. 58 919-924

[75] Landau L D and Lifshift E M 1958 Fluid Mechanics Reading, MA: Addison-Wesley

[76] Hiscock W A and Lindblom L 1985 Phys. Rev. D 31 725; 1987 Phys. Rev. D 353723

[77] Muller I 1967 Z. Physik 198329

[78] Israel W 1976 Ann. Phys., NY 100310

[79] Israel W and Stewart J M 1979 Ann. Phys (N.Y.) 118341

[80] Israel W and Stewart J M 1979 Proc. R. Soc. A 36543

[81] Pavon D, Jou D and Casas-Vázquez J 1982 Ann. Inst. Henri Poincaré Vol. XXXVI 1 79-88

[82] Mubasher Jamil and Muneer Ahmad Rashid 2008 Eur. Phys. J. C 58 111-114

[83] Collaboration 5 year WMAP observations 2008 [arXiv:0803.0732]

[84] Hoyle E 1958. In L'estructure et l'évolution de l'univers Bruxelles, p. 53

[85] Carreta et al, 2000 Astroph. J. 533 215-235

[86] Turner M \& Riess A G 2002 Astrophy. J. 569, 18

[87] Sahni V and Starobinsky A A 2000 Int. J. Mod. Phys. D 9, 373; Sahni V 2004 Lect. Notes Phys. 653, 141; Carroll S M 2001 Living Rev. Relativity 4, 1; Padmanabhan T 2003 Phys. Rep. 380, 235; Peebles P J E \& Ratra B 2003 Rev. Mod. Phys. 75, 559.

[88] Copeland E J, Sami M and Tsujikawa S 2006 Int. J. Mod. Phys. D 15, 1753

[89] Freedman W L et al, 2001 Astrophys. J. 553, 47 